\title{
Schubert problems with respect to osculating flags of stable rational curves
}

\author{
David E Speyer
}

\begin{abstract}
At every point on the rational normal curve, there is an osculating flag. Consider Schubert problems with respect to $r$ such flags, corresponding to $r$ distinct points of the curve. It is natural to consider the $r$ points as parametrized by the moduli space of $r$ distinct points in $\mathbb{P}^{1}$ - the famous $M_{0, r}$. One can then ask to extend the results on Schubert intersections to the compactification $\bar{M}_{0, r}$.

The first part of this paper achieves this goal. We construct a flat, Cohen-Macaulay family $\mathcal{G}(d, n)$ over $\bar{M}_{0, r}$, whose fibers over $M_{0, r}$ are isomorphic to the Grassmannian $G(d, n)$. For any Schubert problem, we construct a flat Cohen-Macaulay subfamily $\mathcal{S}$ of $\mathcal{G}(d, n)$ whose fibers over points of $M_{0, r}$ correspond to the solutions of that Schubert problem with respect to osculating flags.

In the second part of the paper, we investigate the topology of the real points of $\mathcal{S}$, in the case that the Schubert problem has finitely many solutions. We show that this space is a finite cover of $\bar{M}_{0, r}(\mathbb{R})$. We give an explicit CW decomposition of this cover whose faces are indexed by objects from the theory of Young tableaux.
\end{abstract}

\section{Introduction and summary of results}

For a vector space $V$ and a positive integer $d$, we write $G(d, V)$ for the Grassmannian of $d$ dimensional subspaces of $V$. We will write $G(d, n)$ in place of $G\left(d, \mathbb{C}^{n}\right)$.

Consider $2 n-4$ points $z_{1}, \ldots, z_{2 n-4}$ in $\mathbb{P}^{1}$. The Wronski problem is to find rational functions $p(z) / q(z)$ of degree $\leqslant n-1$ which have critical points at the $z_{i}$. Such a rational function is to be considered up to automorphisms of the target space. More intrinsically, let $V$ be the vector space of degree $n-1$ polynomials in $z$. Then $p(z)$ and $q(z)$ span a two-dimensional subspace of $V$, and this subspace is an intrinsic characteristic of the map $z \mapsto p(z) / q(z)$, unchanged by automorphisms of the target. If we consider $\langle p(z), q(z)\rangle$ as a point of the Grassmannian $G(2, V)$, then the condition that $p(z) / q(z)$ has a critical point at $z_{i}$ corresponds to a Schubert condition on $\langle p(z), q(z)\rangle$, and solving the Wronski problem amounts to finding the intersection of $2 n-4$ Schubert conditions in $G(2, n)$.

The group $\mathrm{PGL}_{2}$ acts on $\mathbb{P}^{1}$, moving the points $z_{i}$. This action extends to an action on $G(2, n)$, and to an action on the solutions of the Wronski problem. So it is natural to consider the input to the Wronski problem as $2 n-4$ distinct points $z_{i}$ in $\mathbb{P}^{1}$, modulo the action of $\mathrm{PGL}_{2}$. The space

Received 18 May 2013, accepted in final form 1 July 2013.

2010 Mathematics Subject Classification 14N15 (primary), 14P25 (secondary)

Keywords: Schubert calculus, Wronskian, Shapiro-Shapiro conjecture, monodromy, jeu de tacquin, growth diagram This journal is (C) Foundation Compositio Mathematica 2014. This article is distributed with Open Access under the terms of the Creative Commons Attribution Non-Commercial License, which permits non-commercial reuse, distribution, and reproduction in any medium, provided that the original work is properly cited. For commercial re-use, please contact the Foundation Compositio Mathematica. 


\section{SCHUBERT PROBlems FROM STABLE CURVES}

of $2 n-4$ distinct points in $\mathbb{P}^{1}$ modulo the action of $\mathrm{PGL}_{2}$ is $M_{0,2 n-4}$. Roughly speaking, this paper will extend these ideas to the compactification $\bar{M}_{0,2 n-4}$.

Our construction applies to any Grassmannian and any Schubert problem, not just the ones which arise from the Wronski problem. For $z \in \mathbb{P}^{1}$, let $F_{\bullet}(z)$ be the osculating flag at $z$. This is a complete flag in $\mathbb{C}^{n}$; see Section 2.4 for the definition. For a partition $\lambda$, let $\Omega(\lambda, z)$ be the subvariety of $G(d, n)$ consisting of those $d$-planes which obey Schubert condition $\lambda$ with respect to the flag $F_{\bullet}(z)$. We will review the definitions of Schubert conditions, and conventions for working with partitions, in Sections 2.3 and 2.4. For distinct points $z_{1}, z_{2}, \ldots, z_{r}$ in $\mathbb{P}^{1}$, and partitions $\lambda_{1}, \lambda_{2}, \ldots, \lambda_{r}$, let $\Omega\left(\lambda_{\bullet}, z_{\bullet}\right)$ be $\bigcap_{i=1}^{r} \Omega\left(\lambda_{i}, z_{i}\right)$.

Let $U_{r}$ be the space of $r$ distinct points in $\mathbb{P}^{1}$ and let $\lambda_{1}, \lambda_{2}, \ldots, \lambda_{r}$ be an $r$-tuple of partitions as above. Let $\Omega\left(\lambda_{\bullet}\right) \subset U_{r} \times G(d, n)$ be the variety whose fiber over $\left(z_{1}, \ldots, z_{r}\right) \in U_{r}$ is $\Omega\left(\lambda_{\bullet}, z_{\bullet}\right)$. This variety has been very well studied and the main results are the following:

- For any $\left(z_{1}, \ldots, z_{r}\right)$ in $U_{r}$, the intersection $\bigcap \Omega\left(\lambda_{i}, z_{i}\right)$ is of the expected dimension $d(n-$ $d)-\sum\left|\lambda_{i}\right|$ [EH83, Theorem 2.3]. Combined with the obvious flatness of $\Omega(\lambda)$ over $U_{r}$, and the Cohen-Macaulayness of Schubert varieties [Ram85], this means that $\Omega\left(\lambda_{\bullet}\right)$ is CohenMacaulay, and is flat over $U_{r}$ of relative dimension $d(n-d)-\sum_{i=1}^{r}\left|\lambda_{i}\right|$.

- If $\sum\left|\lambda_{i}\right|=d(n-d)$, then the fiber of $\Omega\left(\lambda_{\bullet}\right)$ over any point of $U_{r}(\mathbb{R})$ is a reduced union of real points [MTV09]. This last result was known as the Shapiro-Shapiro conjecture before it was proved by Mukhin, Tarasov and Varchenko; we will refer to it as the $\boldsymbol{M T} \boldsymbol{V}$ theorem.

These are the results that we will generalize to the case of stable curves.

The group $\mathrm{PGL}_{2}$ acts on $\mathbb{P}^{1}$ and on $G(d, n)$. This gives rise to actions of $\mathrm{PGL}_{2}$ on $U_{r}$ and on $\Omega\left(\lambda_{\bullet}\right) \subset U_{r} \times G(d, n)$. For $r \geqslant 3$, the action of $\mathrm{PGL}_{2}$ on $U_{r}$ is free. We may quotient by this action and get a family $\Omega\left(\lambda_{\bullet}\right) / \mathrm{PGL}_{2} \rightarrow U_{r} / \mathrm{PGL}_{2} \cong M_{0, r}$ which inherits the good properties of the family $\Omega\left(\lambda_{\bullet}\right) \rightarrow U_{r}$. Our goal will be to extend this family to $\bar{M}_{0, r}$.

\subsection{Results}

In all of the following theorems, let $0<d<n$ and let $r \geqslant 3$. All partitions have at most $d$ parts, each of which is at most $n-d$. Our theorems will take place within a flat, projective Cohen-Macaulay family $\mathcal{G}(d, n)$ over $\bar{M}_{0, r}$ whose fibers over $M_{0, r}$ are isomorphic to $G(d, n)$. We will construct this family in Section 3. Our families are similar to those constructed by Eisenbud and Harris [EH87]; we discuss the relation further in Subsection 3.1.

Theorem 1.1. For $\lambda$ a partition with at most $d$ parts, each at most $n-d$, there is a flat, CohenMacaulay closed subfamily $\mathcal{S}_{i}(\lambda)$ of $\mathcal{G}(d, n)$ extending the family $\Omega\left(\lambda, z_{i}\right) / \mathrm{PGL}_{2}$ over $M_{0, r}$. For any partitions $\lambda_{i}$, the intersection $\bigcap_{i=1}^{r} \mathcal{S}_{i}\left(\lambda_{i}\right)$ is Cohen-Macaulay and flat over $\bar{M}_{0, r}$ of dimension $d(n-d)-\sum\left|\lambda_{i}\right|$. If $d(n-d)-\sum\left|\lambda_{i}\right|<0$, then this intersection is empty.

We write $\mathcal{S}\left(\lambda_{\bullet}\right)$ for $\bigcap_{i=1}^{r} \mathcal{S}_{i}\left(\lambda_{i}\right)$. We now describe the irreducible components of the fibers of $\mathcal{S}\left(\lambda_{\bullet}\right)$. Let $\left(C, z_{\bullet}\right)$ be a stable genus 0 curve with $r$ marked points $z_{1}, \ldots, z_{r}$. Let $C_{1}, \ldots, C_{v}$ be the irreducible components of $C$ and let $w_{1}, \ldots, w_{v-1}$ be the nodes of $C$. We will say that $x \in C_{j}$ is a special point if it is either a marked point, or a node. For a partition $\mu=\left(\mu_{1}, \mu_{2}, \ldots, \mu_{d}\right)$, let $\mu^{C}$ be the complementary partition $\left(n-d-\mu_{d}, n-d-\mu_{d-1}, \ldots, n-d-\mu_{1}\right)$. Consider the following combinatorial data. For each component $C_{j}$ of $C$, and each special point $x \in C_{j}$, assign a partition $\nu\left(C_{j}, x\right)$, obeying the following conditions:

(1) If $z_{i}$ lies in $C_{j}$, we have $\nu\left(C_{j}, z_{i}\right)=\lambda_{i}$. 


\section{DAVID E SPEYER}

(2) If $C_{j}$ and $C_{k}$ meet at the node $w$, then $\nu\left(C_{j}, w\right)=\nu\left(C_{k}, w\right)^{C}$.

We refer to such a $\nu$ as a node labeling. Given the stable curve $\left(C, z_{\bullet}\right)$ and this combinatorial data, let $\mathcal{T}\left(C, z_{\bullet}, \nu_{\bullet}\right)$ be the variety $\prod_{j=1}^{v} \Omega\left(\nu\left(C_{j}, x_{\bullet}\right), x_{\bullet}\right)$, where $x_{\bullet}$ runs over the special points of $C_{j}$. Let $\mathcal{T}\left(C, z_{\bullet}\right)=\bigsqcup_{\nu_{\bullet}} \mathcal{T}\left(C, z_{\bullet}, \nu_{\bullet}\right)$, where the union runs over all node labelings.

Theorem 1.2. The irreducible components of the fiber of $\mathcal{S}\left(\lambda_{\bullet}\right)$ over $\left(C, z_{\bullet}\right)$ are isomorphic to the irreducible components of $\mathcal{T}\left(C, z_{\bullet}\right)$.

We do not attempt to describe how the components of $\mathcal{S}\left(\lambda_{\bullet}\right)$ meet.

For many possible maps $\nu$, the product $\prod_{j=1}^{v} \Omega\left(\nu\left(C_{j}, x_{\bullet}\right), x_{\bullet}\right)$ is empty. In particular, if $\sum_{x \in C_{j}}\left|\nu\left(C_{j}, x\right)\right|>d(n-d)$ for some component $j$, then we get no components for this $\nu_{\bullet}$, so we can restrict to the case that $\sum_{x \in C_{j}}\left|\nu\left(C_{j}, x\right)\right| \leqslant d(n-d)$ for every $C_{j}$. In the case that $\sum\left|\lambda_{i}\right|=d(n-d)$, this can be made into an equality:

Proposition 1.3. Let $\sum\left|\lambda_{i}\right|=d(n-d)$ and let $\nu$ be a node labeling. If $\prod_{j=1}^{v} \Omega\left(\nu\left(C_{j}, x_{\bullet}\right), x_{\bullet}\right)$ is nonempty then $\sum_{x \in C_{j}}\left|\nu\left(C_{j}, x\right)\right|=d(n-d)$ for every component $C_{j}$.

In particular, when $\sum\left|\lambda_{i}\right|=d(n-d)$ and we are over a real point of $\bar{M}_{0, r}$, we can make an especially nice statement.

THEOREM 1.4. If $\sum\left|\lambda_{i}\right|=d(n-d)$, then the fiber of $\mathcal{S}\left(\lambda_{\bullet}\right)$ over $\bar{M}_{0, r}(\mathbb{R})$ is a reduced union of real points. In other words, $\mathcal{S}\left(\lambda_{\bullet}\right)(\mathbb{R})$ is an unbranched cover of $\bar{M}_{0, r}(\mathbb{R})$.

The preceding results are proved in Section 3. The rest of the paper analyzes the topology of $\mathcal{S}\left(\lambda_{\bullet}\right)(\mathbb{R})$ when $\sum\left|\lambda_{i}\right|=d(n-d)$.

There is a $C W$-decomposition of $\bar{M}_{0, r}(\mathbb{R})$, which we detail in Section 5 . The maximal faces are indexed by the $(r-1) ! / 2$ dihedral symmetry classes of circular orderings of $\{1, \ldots, r\}$. The vertices of this $C W$-decomposition are indexed by trivalent trees whose leaves are labeled by $\{1,2, \ldots, r\}$, and correspond to stable curves whose dual graphs are those trees.

We will describe the pull-back of this $C W$-decomposition to the cover $\mathcal{S}\left(\lambda_{\bullet}\right)(\mathbb{R})$. We will call a vertex of the $C W$-decomposition a caterpillar point if every component of the curve contains at least one marked point (see Figure 6). See Sections 6 and 9 for the full definitions underlying the next theorems; readers experienced with tableaux combinatorics may be able to guess the definition of a cylindrical growth diagram by glancing at Figure 4.

We first describe the case where all the partitions are a single box.

Theorem 1.5. Let $r=d(n-d)$ and let all the $\lambda_{i}$ be the partition (1). We describe the pullback of the $C W$-decomposition of $\bar{M}_{0, r}(\mathbb{R})$ to $\mathcal{S}\left(\lambda_{\bullet}\right)(\mathbb{R})$. The maximal faces of this $C W$-decomposition lying over a given maximal face $\sigma$ of $\bar{M}_{0, r}(\mathbb{R})$ are indexed by cylindrical growth diagrams. Over a caterpillar point of $\bar{M}_{0, r}$, the vertices of $\mathcal{S}\left(\lambda_{\bullet}\right)(\mathbb{R})$ are indexed by standard Young tableaux of shape $(n-d)^{d}$. Let $\sigma$ be a maximal face of $\bar{M}_{0, r}$ and $\tau$ a maximal face of $\mathcal{S}\left(\lambda_{\bullet}\right)(\mathbb{R})$ lying above it; let $\gamma$ be the cylindrical growth diagram labeling $\tau$. By following different paths through $\gamma$, we read off the tableaux labeling the vertices of $\tau$ over the caterpillar points of $\sigma$.

We also describe the adjacencies between these maximal faces (Proposition 7.6).

Transforming from one path to another in a growth diagram changes the tableau by promotion and other related operations. Our result is thus related to results of Purbhoo [Pur10], [Pur13]. Purbhoo indexes fibers of $\Omega((1),(1), \cdots,(1))$ over certain points of $U_{r}$ by standard Young 


\section{SCHUBERT PROBlEMS FROM STABLE CURVES}

tableaux and relates monodromy between these points to promotion. Purbhoo works with $U_{r}$; we hope to demonstrate that working over $\bar{M}_{0, r}$ provides cleaner results and proofs.

We now generalize this result to arbitrary partitions obeying $\sum\left|\lambda_{i}\right|=d(n-d)$. For a standard Young tableau $T$ of shape $(n-d)^{d}$, let $\alpha_{s}$ be the subskew tableau with entries $1+\sum_{i=1}^{s-1}\left|\lambda_{i}\right|$, $2+\sum_{i=1}^{s-1}\left|\lambda_{i}\right|, \ldots,\left|\lambda_{s}\right|+\sum_{i=1}^{s-1}\left|\lambda_{i}\right|$. For another standard Young tableau $T^{\prime}$ of the shape $(n-d)^{d}$, let $\alpha_{1}^{\prime}, \ldots, \alpha_{r}^{\prime}$ be the corresponding subskew tableau. We will say that $T$ and $T^{\prime}$ are dual equivalent if each $\alpha_{i}$ is dual equivalent to $\alpha_{i}^{\prime}$. (This definition implicitly depends on the cardinality of the $\lambda_{i}$.)

TheOREM 1.6. Let the $\lambda_{i}$ obey $\sum_{i=1}^{r}\left|\lambda_{i}\right|=d(n-d)$. We give a $C W$-decomposition of $\mathcal{S}\left(\lambda_{\bullet}\right)(\mathbb{R})$ which maps down to the $C W$-decomposition of $\bar{M}_{0, d(n-d)}(\mathbb{R})$. The points of $\mathcal{S}\left(\lambda_{\bullet}\right)(\mathbb{R})$ over caterpillar points of $\bar{M}_{0, r}$ are indexed by dual equivalence classes of standard Young tableaux for which $\alpha_{i}$ rectifies to a tableau of shape $\lambda_{i}$. The maximal faces of $\mathcal{S}\left(\lambda_{\bullet}\right)(\mathbb{R})$ lying over a maximal face $\sigma$ of $\bar{M}_{0, r}(\mathbb{R})$ are indexed by dual equivalence classes of cylindrical growth diagrams with shape $\left(\lambda_{1}, \ldots, \lambda_{r}\right)$. Let $\sigma$ be a maximal face of $\bar{M}_{0, r}$ and $\tau$ a maximal face of $\mathcal{S}\left(\lambda_{\bullet}\right)(\mathbb{R})$ lying above it; let $\gamma$ be the dual equivalence class of cylindrical growth diagrams corresponding to $\tau$. By following different paths through $\gamma$, we read off the dual equivalence classes of tableaux labeling the vertices of $\tau$ over the caterpillar points of $\sigma$.

I originally intended to conclude with an appendix discussing the relations between dual equivalence classes of growth diagrams, cartons [TY08], hives and the octahedron recurrence [KTW04], [HK06], but it has grown too lengthy and will be a separate paper.

\subsection{Acknowledgements}

This paper has developed over several years, and benefited from conversations with many mathematicians. Particularly, I would like to thank Allen Knutson, Joel Kamnitzer, Kevin Purbhoo and Frank Sottile. My thanks also to the referee for several helpful suggestions. Some of the research in this paper was done while I was supported by a research fellowship from the Clay Mathematical Institute.

\section{Notation and background}

\subsection{Combinatorial generalities}

We write $[n]$ for $\{1,2, \ldots, n\}$. For any finite set $S$, we write $\left(\begin{array}{l}S \\ d\end{array}\right)$ for the collection of $d$-element subsets of $S$. We will sometimes abbreviate $S \cup\{i, j\}$ to $S i j$ and $S \backslash\{k\}$ to $S \backslash k$.

\subsection{Trees and stable curves}

A tree is a finite, connected, contractable graph. For a tree $\Gamma$, a leaf is a vertex of degree 1; we call a vertex internal if it has degree $>1$. We write $\Gamma^{\circ}$ for the set of internal vertices. A tree is trivalent if every internal vertex has degree 3. We define an internal edge to be an edge between two internal vertices, and write $\Gamma_{1}^{\circ}$ for the set of internal edges.

Let $C$ be a nodal curve with $n$ distinct marked smooth points. The corresponding $\boldsymbol{d u a l}$ graph has a vertex for each component of $C$, and a vertex for each marked point. For every node, lying on components $D_{1}$ and $D_{2}$, there is an edge joining the vertices corresponding to $D_{1}$ and $D_{2}$. Additionally, for every marked point $x$ contained in a component $D$, there is an edge between the vertices corresponding to $x$ and $D$.

A nodal curve as above is called a stable genus 0 curve with $n$ marked points if the 


\section{DAVID E SPEYER}
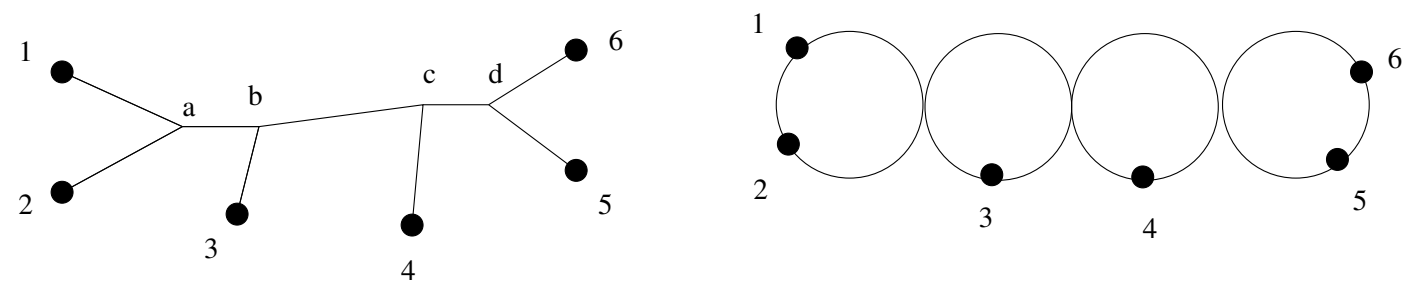

Figure 1. A stable curve and its dual tree

dual graph is a tree with no vertices of degree 2, and where every degree 1 vertex corresponds to a marked point. In this case, we will write dual tree rather than dual graph. There is a moduli space of stable genus 0 curves with $n$ marked points, denoted by $\bar{M}_{0, n}$. It is stratified according to the combinatorial type of the dual tree. There is one largest stratum called $M_{0, n}$, where $C$ has only one component. There are $(2 n-1)(2 n-3) \cdots 3 \cdot 1$ zero dimensional strata, corresponding to the trivalent trees with vertices labeled by $[n]$. Figure 1 shows a stable curve and the corresponding dual tree. This is one of the 105 trivalent trees with leaves labeled by [6]; the letters labeling the internal vertices play no role for now.

Occasionally, it is convenient to label the nodes of a stable curve with a finite set $A$ other then $[n]$. We write $\bar{M}_{0, A}$ in this case.

\subsection{Partitions and Schubert calculus}

We often want to work with partitions with at most $d$ parts, each at most $n-d$. The set of all such partitions will be denoted by $\Lambda$, or $\Lambda(d, n)$ if necessary. We denote the partition $(0,0,0, \ldots)$ by $\emptyset,(1,0,0, \ldots)$ by $\square$ and $(n-d, n-d, \ldots, n-d)$ by $\boxplus$. As mentioned in the introduction, we set $\left(\lambda_{1}, \lambda_{2}, \ldots, \lambda_{d}\right)^{C}=\left(n-d-\lambda_{d}, \cdots, n-d-\lambda_{2}, n-d-\lambda_{1}\right)$.

For partitions $\lambda_{1}, \lambda_{2}, \ldots, \lambda_{s}$ and $\mu$, with $|\mu|=\sum\left|\lambda_{i}\right|$, we write $c_{\lambda_{1} \lambda_{2} \cdots \lambda_{s}}^{\mu}$ for the LittlewoodRichardson coefficient. We will often use the identity

$$
c_{\lambda_{1} \lambda_{2} \cdots \lambda_{r}}^{\mathrm{\#}}=c_{\lambda_{1} \lambda_{2} \cdots \lambda_{r-1}}^{\lambda_{r}^{C}}
$$

For any two partitions $\mu, \nu$ with $\mu \subseteq \nu$, let $\operatorname{SYT}(\nu / \mu)$ be the set of chains of partitions which grow from $\mu$ to $\nu$ adding one box at a time. For $T \in \operatorname{SYT}(\nu / \mu)$, we write $\operatorname{shape}(T)=\nu / \mu$. If shape $(T)=\lambda / \emptyset$, we say that $T$ is of straight shape and we also write shape $(T)=\lambda$. We adopt the abbreviations $\operatorname{SYT}(\lambda)$ for $\operatorname{SYT}(\lambda / \emptyset)$, and $\operatorname{SYT}(d, n)$ for $\operatorname{SYT}(\boxplus)$.

Let $n-d \geqslant \lambda_{1} \geqslant \lambda_{2} \geqslant \cdots \geqslant \lambda_{d} \geqslant 0$ be a partition in $\Lambda(d, n)$, where we pad by zeroes as necessary. Then we define $I(\lambda)$ to be the subset $\left(\lambda_{d}+1, \lambda_{d-1}+2, \ldots, \lambda_{1}+d\right)$ of $[n]$. This is a bijection between $\Lambda$ and $\left(\begin{array}{c}{[n]} \\ d\end{array}\right)$.

\subsection{Schubert varieties}

For a flag $0=F_{0} \subset F_{1} \subset F_{2} \subset \cdots \subset F_{n-1} \subset F_{n}=\mathbb{C}^{n}$, with $\operatorname{dim} F_{i}=i$, and a partition $\lambda \in \Lambda$, we say that a $d$-plane $V$ in $\mathbb{C}^{n}$ obeys the Schubert condition $\lambda$ with respect to $F_{\bullet}$ if $\operatorname{dim}\left(V \cap F_{i}\right) \geqslant \#(I(\lambda) \cap\{n-i+1, n-i+2, \ldots, n\})$. The variety of $d$-planes obeying Schubert condition $\lambda$ with respect to $F_{\bullet}$ is denoted by $\Omega\left(\lambda, F_{\bullet}\right)$. It has codimension $|\lambda|$. The cohomology class $\left[\Omega\left(\lambda, F_{\bullet}\right)\right] \in H^{2|\lambda|}(G(d, n))$ is independent of $F_{\bullet}$, so we will drop the flag from our notation 


\section{Schubert PRoblems From STABle CURVES}

when speaking only of cohomology classes. We have

$$
\prod_{i=1}^{s}\left[\Omega\left(\lambda_{i}\right)\right]=\sum c_{\lambda_{1} \cdots \lambda_{s}}^{\mu}[\Omega(\mu)] .
$$

If $\sum\left|\lambda_{i}\right|=d(n-d)$, then $\prod\left[\Omega\left(\lambda_{i}\right)\right]$ is $c_{\lambda_{1} \lambda_{2} \cdots \lambda_{r}}^{\text {曲 }}$ times the fundamental class. If $F_{1}, F_{2}, \ldots, F_{r}$ are generic flags, then $\bigcap_{i=1}^{r} \Omega\left(\lambda_{i}, F_{i}\right)$ consists of $c_{\lambda_{1} \lambda_{2} \cdots \lambda_{r}}^{\text {m }}$ reduced points.

For $z \in \mathbb{C}^{1}$, let $F_{\bullet}(z)$ be the flag spanned by the top rows of the matrix

$$
\left(\begin{array}{ccccc}
1 & z & z^{2} & \cdots & z^{n} \\
0 & 1 & 2 z & \cdots & n z^{n-1} \\
0 & 0 & 2 & \cdots & n(n-1) z^{n-2} \\
& & & \ddots & \\
0 & 0 & 0 & \cdots & n !
\end{array}\right) .
$$

We can extend this continuously to any $z \in \mathbb{P}^{1}$ by letting $F_{\bullet}(\infty)$ be spanned by the top rows of

$$
\left(\begin{array}{ccccc}
0 & \cdots & 0 & 0 & 1 \\
0 & \cdots & 0 & 1 & 0 \\
0 & \cdots & 1 & 0 & 0 \\
& \cdot \cdot & & & \\
1 & 0 & 0 & \cdots & 0
\end{array}\right)
$$

We shorten $\Omega\left(\lambda, F_{\bullet}(z)\right)$ to $\Omega(\lambda, z)$. We give an explicit formula for $\Omega(\square, z)$.

Proposition 2.1. Let $z \in \mathbb{P}^{1}$ and let $\left(p_{I}\right)$ be a point of $G(d, n)$, given as a list of $\left(\begin{array}{l}n \\ d\end{array}\right)$ Plücker coordinates $p_{I}$. For $I \in\left(\begin{array}{c}{[n]} \\ d\end{array}\right)$, define

$$
\Delta(I)=\prod_{\substack{a, b \in I \\
a<b}}(b-a) \text { and } \ell(I)=\sum_{i \in I} i-\left(\begin{array}{l}
d \\
2
\end{array}\right) .
$$

Then $\left(p_{I}\right)$ obeys the Schubert condition $\square$ with respect to $F_{\bullet}(z)$ if and only if

$$
\sum_{I \in\left(\begin{array}{c}
{[n]} \\
d
\end{array}\right)} \Delta(I) \cdot P_{I} \cdot(-z)^{d(n-d)-\ell(I)}=0 .
$$

Proof. See [Pur10, Proposition 2.3]. Purbhoo's conventions are related to ours by the involution $z \mapsto-z^{-1}$ of $\mathbb{P}^{1}$.

These flags have surprising transversality properties:

THEOREM 2.2 ([EH83]). For any distinct points $z_{1}, z_{2}, \ldots, z_{r}$ in $\mathbb{C P}^{1}$, and any partitions $\lambda_{1}, \lambda_{2}, \ldots, \lambda_{r}$, the intersection $\bigcap \Omega\left(\lambda_{i}, z_{i}\right)$ has codimension $\sum\left|\lambda_{i}\right|$. If the product $\prod\left[\Omega\left(\lambda_{i}\right)\right]$ in $H^{*}(G(d, n))$ is zero, then this intersection is empty.

TheOREM 2.3 ([MTV09]). For any distinct points $z_{1}, z_{2}, \ldots, z_{r}$ in $\mathbb{R P}^{1}$ and any partitions $\lambda_{1}$, $\lambda_{2}, \ldots, \lambda_{r}$ with $\sum\left|\lambda_{i}\right|=d(n-d)$, the intersection $\bigcap \Omega\left(\lambda_{i}, z_{i}\right)$ consists of $c_{\lambda_{1} \lambda_{2} \cdots \lambda_{r}}$ reduced points.

\subsection{Lemmas about Cohen-Macaulay varieties}

Theorem 2.4 ([Bri02, Lemma 1]). Let $Z$ be smooth and let $X$ and $Y$ be subvarieties of $Z$ which are Cohen-Macaulay and pure of codimension $s$ and $t$ respectively. If $Y \cap Z$ has codimension $s+t$, then $Y \cap Z$ is Cohen-Macaulay. 


\section{DAVID E SPEYER}

Theorem 2.5 ([Mat86, Theorem 23.1]). Let $B$ be smooth, $X$ Cohen-Macaulay, and $\pi: X \rightarrow B$ surjective with all fibers of the same dimension. Then $\pi$ is flat and the fibers of $\pi$ are CohenMacaulay.

We will often use these theorems together:

Corollary 2.6. Let $B$ be smooth, let $Z \rightarrow B$ be a smooth map, and let $X$ and $Y$ be subvarieties of $Z$ which are Cohen-Macaulay, flat over $B$, and have codimensions $s$ and $t$ in $Z$. If the fibers of $X \cap Y$ have codimension $s+t$, then these fibers are Cohen-Macaulay, and $X \cap Y$ is flat over $B$.

\section{The families $\mathcal{G}(d, n)$ and $\mathcal{S}\left(\lambda_{\bullet}\right)$}

In this section, we construct the families $\mathcal{G}(d, n)$ and $\mathcal{S}\left(\lambda_{\bullet}\right)$ over $\bar{M}_{0, r}$. We take $0<d<n$ and $r \geqslant 3$. This construction will involve many varieties which are not seen again outside this section.

Let $T$ be a set with three elements. Let $\mathbb{C}_{T}^{2}$ be the two-dimensional vector space of functions $f: T \rightarrow \mathbb{C}$, modulo the subspace spanned by $(1,1,1)$. Let $\mathbb{P}_{T}^{1}$ be the projective space of lines in $\mathbb{C}^{T}$. Note that $\mathbb{P}_{T}^{1}$ has three points $z(t)$ canonically labeled by $t \in T$, corresponding to the standard basis of $\mathbb{C}_{T}^{2}$. Let $V_{T}=\operatorname{Sym}^{n-1} \mathbb{C}_{T}^{2}$ and let $G(d, n)_{T}$ be the Grassmannian of $d$-dimensional subspaces of $V_{T}$. We have the Segre embedding $\mathbb{P}_{T}^{1} \hookrightarrow \mathbb{P}\left(V_{T}\right)$, induced by sending $v \in \mathbb{C}_{T}^{2}$ to $v^{\otimes(n-1)}$ in $V_{T}$. For $z \in \mathbb{P}_{T}^{1}$, we can define a flag $F_{\bullet}(z)$ in $V_{T}$ as before, and we can then define Schubert varieties $\Omega(\lambda, z)_{T} \subseteq G(d, n)_{T}$ with respect to that flag. For $t \in T$, we abbreviate $\Omega(\lambda, z(t))_{T}$ to $\Omega(\lambda, t)_{T}$

Let $Q=\left\{p_{1}, p_{2}, q_{1}, q_{2}\right\}$ be a set with four elements. Let $C$ be a genus 0 curve with four distinct marked points $z\left(p_{1}\right), z\left(p_{2}\right), z\left(q_{1}\right), z\left(q_{2}\right)$. Let $T_{1}=\left(p_{1}, p_{2}, q_{1}\right)$ and let $T_{2}=\left(p_{1}, p_{2}, q_{2}\right)$. Then we have unique isomorphisms $\mathbb{P}_{T_{1}}^{1} \cong C \cong \mathbb{P}_{T_{2}}^{1}$, taking the labeled points of the $\mathbb{P}_{T_{i}}^{1}$ to the labeled points of $C$. Let $\phi\left(C, z\left(p_{1}\right), z\left(p_{2}\right), z\left(q_{1}\right), z\left(q_{2}\right)\right): \mathbb{P}_{T_{1}}^{1} \rightarrow \mathbb{P}_{T_{2}}^{1}$ be the composite isomorphism. This induces an isomorphism from $G(d, n)_{T_{1}}$ to $G(d, n)_{T_{2}}$ which we'll denote by $\phi\left(C, z\left(p_{1}\right), z\left(p_{2}\right), z\left(q_{1}\right), z\left(q_{2}\right)\right)_{*}$. Define $\mathcal{X}_{0}\left(p_{1}, p_{2} ; q_{1}, q_{2}\right)$ to be the closed subvariety of $M_{0, Q} \times G(d, n)_{T_{1}} \times G(d, n)_{T_{2}}$ whose fiber over $\left(C, z\left(p_{1}\right), z\left(p_{2}\right), z\left(q_{1}\right), z\left(q_{2}\right)\right)$ is the graph of the map $\phi\left(C, z\left(p_{1}\right), z\left(p_{2}\right), z\left(q_{1}\right), z\left(q_{2}\right)\right)_{*}$. Let $\mathcal{X}\left(p_{1}, p_{2} ; q_{1}, q_{2}\right)$ be the closure of $\mathcal{X}_{0}\left(p_{1}, p_{2} ; q_{1}, q_{2}\right)$ in $\bar{M}_{0, Q} \times G(d, n)_{T_{1}} \times G(d, n)_{T_{2}}$.

In the next proposition and its proof, we abbreviate $\mathcal{X}_{0}\left(p_{1}, p_{2} ; q_{1}, q_{2}\right)$ to $\mathcal{X}_{0}$ and $\mathcal{X}\left(p_{1}, p_{2} ; q_{1}, q_{2}\right)$ to $\mathcal{X}$.

Proposition 3.1. Over every point of $M_{0, Q}$, the fiber of $\mathcal{X}$ is isomorphic to $G(d, n)$. Over the point of $\bar{M}_{0, Q}$ where $q_{1}$ and $q_{2}$ collide, the fiber is also isomorphic to $G(d, n)$.

Over the two points of $\bar{M}_{0, Q}$ where one of the $p$ 's collides with one of the $q$ 's, the fiber of $\mathcal{X}$ is the reduced union of $\left(\begin{array}{l}n \\ d\end{array}\right)$ irreducible components, indexed by partitions $\nu \in \Lambda$. More specifically, when $p_{1}$ and $q_{1}$ collide, the fiber of $\mathcal{X}$ is the reduced union $\bigcup_{\nu} \Omega\left(\nu, z\left(p_{2}\right)\right) \times \Omega\left(\nu^{C}, z\left(p_{1}\right)\right)$. When $p_{1}$ and $q_{2}$ collide, the fiber of $\mathcal{X}$ is the reduced union $\bigcup_{\nu} \Omega\left(\nu, z\left(p_{1}\right)\right) \times \Omega\left(\nu^{C}, z\left(p_{2}\right)\right)$

The total space of $\mathcal{X}$ is reduced and Cohen-Macaulay; $\mathcal{X} \rightarrow \bar{M}_{0, Q}$ is flat.

Proof. Over $M_{0, Q}$, the fibers of $\mathcal{X}$ are graphs of maps $G(d, n)_{T_{1}} \rightarrow G(d, n)_{T_{2}}$, so they are isomorphic to $G(d, n)$. When $q_{1}$ collides with $q_{2}$, the isomorphism $\phi$ becomes the "identity map", taking the labeled points of $\mathbb{P}_{T_{1}}^{1}$ to the labeled points of $\mathbb{P}_{T_{2}}^{1}$, so the fiber of $\mathcal{X}$ is again the graph of a map. Thus, all of the interest is near the two remaining boundary points of $\bar{M}_{0, Q}$.

We switch to explicit coordinates. Let $z\left(p_{1}\right), z\left(p_{2}\right)$ and $z\left(q_{1}\right)$ be at $0, \infty$ and 1 , respectively, 


\section{SCHUBERT PROBlems FROM STABLE CURVES}

and let $\tau$ be the position of $z\left(q_{2}\right)$. We will study what happens as $\tau \rightarrow 0$; the case where $\tau \rightarrow \infty$ is similar. The map $\mathbb{P}_{T_{1}}^{1} \rightarrow \mathbb{P}_{T_{2}}^{1}$ is given by $u \mapsto \tau u$.

Brion [Bri02, Section 2] constructs a subvariety $\mathcal{Y}$ of $\mathbb{C}^{n-1} \times G(d, n) \times G(d, n)$, flat over $\mathbb{C}^{n-1}$, whose fiber over $(1,1, \ldots, 1)$ is the diagonal of $G(d, n) \times G(d, n)$ and whose fiber over $(0,0, \ldots, 0)$ is the union of Schubert varieties described above. Our family is precisely the restriction of Brion's family to the curve $\gamma \subset \mathbb{C}^{n-1}$ parametrized by $\left(\tau, \tau^{2}, \cdots, \tau^{n-1}\right)$. Since $\mathcal{Y}$ is flat, so is our subvariety, and it has the described fibers. The curve $\gamma$ is a complete intersection in $\mathbb{C}^{n-1}$, cut out by the equations $x_{k}=x_{1}^{k}$, for $2 \leqslant k \leqslant n-1$. Since $\mathcal{Y}$ is flat over $\mathbb{C}^{n-1}$, the regular sequence $\left(x_{2}-x_{1}^{2}, x_{3}-x_{1}^{3}, \ldots, x_{n-1}-x_{1}^{n-1}\right)$ on $\mathbb{C}^{n-1}$ pulls back to a regular sequence on $\mathcal{Y}$. The quotient of a Cohen-Macaulay variety by a regular sequence is Cohen-Macaulay, so the restriction of $\mathcal{Y}$ to $\gamma$ is Cohen-Macaulay again.

We will frequently engage in the following abuse of notation: With the above notation, let $Q \subset[r]$, and let $T_{3}, T_{4}, \ldots, T_{m}$ be three-element subsets of $[r]$. Then we have a natural projection $\bar{M}_{0, r} \times \prod_{i=1}^{m} G(d, n)_{T_{i}} \rightarrow \bar{M}_{0, Q} \times G(d, n)_{T_{1}} \times G(d, n)_{T_{2}}$. We will use the notation $\mathcal{X}\left(p_{1}, p_{2} ; q_{1}, q_{2}\right)$ to also refer to the preimage of $\mathcal{X}\left(p_{1}, p_{2} ; q_{1}, q_{2}\right)$ inside $\bar{M}_{0, r} \times \prod_{i=1}^{m} G(d, n)_{T_{i}}$. When necessary for clarity, we will say that we are considering $\mathcal{X}\left(p_{1}, p_{2} ; q_{1}, q_{2}\right)$ inside $\bar{M}_{0, r} \times \prod_{i=1}^{m} G(d, n)_{T_{i}}$. Similarly, for $T \in\left(\begin{array}{c}{[r]} \\ 3\end{array}\right)$ and $t \in T$, we will write $\Omega(\lambda, t)_{T}$ for the preimage of $\Omega(\lambda, t)_{T}$ under the $\operatorname{map} \bar{M}_{0, r} \times \prod_{i=1}^{m} G(d, n)_{T_{i}} \rightarrow G(d, n)_{T}$.

We can now define the families $\mathcal{G}(d, n)$ and $\mathcal{S}\left(\lambda_{\bullet}\right)$. They are both defined as closed subfamilies of $\bar{M}_{0, r} \times \prod_{T \in\left(\begin{array}{c}{[n]} \\ 3\end{array}\right)} G(d, n)_{T}$. Namely,

$$
\mathcal{G}(d, n)=\bigcap_{p_{1}, p_{2}, q_{1}, q_{2} \in[r]} \mathcal{X}\left(p_{1}, p_{2} ; q_{1}, q_{2}\right) \text { and } \mathcal{S}\left(\lambda_{\bullet}\right)=\mathcal{G}(d, n) \cap \bigcap_{t \in T \in\left(\begin{array}{c}
{[r]} \\
3
\end{array}\right)} \Omega\left(\lambda_{t}, t\right)_{T} .
$$

We recall the promises we have made about $\mathcal{G}(d, n)$ :

Theorem 3.2. The family $\mathcal{G}(d, n)$ defined above is Cohen-Macaulay. It is projective and flat of relative dimension $d(n-d)$ over $\bar{M}_{0, r}$. The fibers of $\mathcal{G}(d, n)$ over $M_{0, r}$ are all isomorphic to $G(d, n)$.

The remainder of this section consists of the proofs of Theorems 3.2, 1.1 and 1.4. We begin by checking that the fibers over $M_{0, r}$ are as claimed.

Proposition 3.3. Over $M_{0, r}$, the fibers of $\mathcal{G}(d, n)$ are all isomorphic to $G(d, n)$, embedded in $\prod G(d, n)_{T}$ with the cohomology class of the small diagonal. The fiber of $\mathcal{S}\left(\lambda_{\bullet}\right)$ over a point $\left(z_{1}, z_{2}, \ldots, z_{r}\right)$ of $M_{0, r}$ is isomorphic to $\Omega\left(\lambda_{\bullet}, z_{\bullet}\right)$, inside the $G(d, n)$ of the previous sentence.

Proof. Fix a point $\left(z_{1}, \ldots, z_{r}\right)$ and consider the fibers over it. All of the varieties $\mathcal{X}\left(p_{1}, p_{2} ; q_{1}, q_{2}\right)$ are graphs of isomorphisms between two of the $G(d, n)_{T}$ 's, and all these isomorphisms are compatible. So the fiber of $\mathcal{G}(d, n)$ over $\left(z_{\bullet}\right)$ consists of those points that are identified by all of these compatible isomorphisms, and is thus isomorphic to $G(d, n)$. Imposing the Schubert conditions in addition imposes those Schubert conditions within this $G(d, n)$.

All the claims in Theorems 3.2, 1.1 and 1.4 are local on $\bar{M}_{0, r}$, so it is enough to prove them restricted to an open cover of $\bar{M}_{0, r}$. We will now introduce terminology to describe the open cover.

Fix a trivalent tree $\Gamma$ with leaves labeled by $[r]$. We will use $\Gamma$ to define an open subset $U(\Gamma)$ of $\bar{M}_{0, r}$. For a stable curve $\left(C, z_{\bullet}\right)$, let $\Delta$ be the dual tree of $C$. We define the point $\left(C, z_{\bullet}\right)$ to be 


\section{DAVID E SPEYER}

in $U(\Gamma)$ if there is a continuous map $\phi: \Gamma \rightarrow \Delta$ which takes leaves to leaves, preserving labels, takes internal vertices to internal vertices and takes edges either to edges or to internal vertices. Another way to state this condition is that $\left(C, z_{\bullet}\right)$ is in $U(\Gamma)$ if and only if, for every edge $e$ of $\Delta$, there is an edge $e^{\prime}$ of $\Gamma$ so that removing the interiors of $e$ and $e^{\prime}$ from their respective trees partitions $[r]$ into the same two pieces.

Every point of $\bar{M}_{0, r}$ is in some $U(\Gamma)$. Let $\mathcal{G}(d, n)(\Gamma)$ and $\mathcal{S}\left(\lambda_{\bullet}\right)(\Gamma)$ be the open subsets of the corresponding families living over $U(\Gamma)$.

For any $T \in\left(\begin{array}{c}{[r]} \\ 3\end{array}\right)$, let $v(T)$ be the unique internal vertex of $\Gamma$ such that the elements of $T$ label leaves in three different components of $\Gamma \backslash v(T)$. In Figure 1, for example, $v(\{1,3,5\})=b$ and $v(\{1,5,6\})=d$.

Let $v$ be an internal vertex of $\Gamma$. Define $G(d, n)_{v}$ to be the intersection inside $U(\Gamma) \times$ $\prod_{T: v(T)=v} G(d, n)_{T}$ of those $\mathcal{X}\left(p_{1}, p_{2} ; q_{1}, q_{2}\right)$ 's for which $v=v\left(p_{1}, p_{2}, q_{1}\right)=v\left(p_{1}, p_{2}, q_{2}\right)$.

Proposition 3.4. For every $T$ with $v(T)=v$, the projection $G(d, v)_{v} \rightarrow G(d, n)_{T} \times U(\Gamma)$ is an isomorphism.

Proof. $G(d, n)_{v}$ is the intersection of many varieties of the form $\mathcal{X}\left(p_{1}, p_{2} ; q_{1}, q_{2}\right)$, each of which is the graph of an isomorphism between one $G(d, n)_{T}$ and another $G(d, n)_{T^{\prime}}$, and all these isomorphisms are compatible.

We will want to make computations in fibers of families over $U(\Gamma)$. We now introduce suitable notation for this purpose. Fix $u \in U(\Gamma)$. For $Z \rightarrow U(\Gamma)$, let $Z(u)$ be the fiber over $u$. Moreover, if $Z_{1}, \ldots, Z_{k}$ are families over $U(\Gamma)$, then we let $\prod_{i=1}^{k} Z_{i}$ denote the product over $U(\Gamma)$. For example, $\prod_{i=1}^{3} Z_{i}$ means $Z_{1} \times_{U(\Gamma)} Z_{2} \times_{U(\Gamma)} Z_{3}$.

Let $C$ be the stable curve corresponding to $u$ and let $\Delta$ be the dual tree. For an internal vertex $v$ of $\Delta$, let $C(v)$ be the corresponding component of $C$. Let $\mathbb{C}^{2}(v)$ be a two-dimensional vector space with a chosen identification $\mathbb{P}\left(\mathbb{C}^{2}(v)\right) \cong C(v)$. Let $V(v)=\operatorname{Sym}^{n-1} \mathbb{C}^{2}(v)$ and let $G(d, n)(v)$ be the Grassmannian of $d$-planes in $V(v)$.

For an edge $e$ of $\Delta$, with endpoint $v \in \Delta^{\circ}$, let $p(v, e)$ be the marked point of $C(v)$ corresponding to $e$. Let $\Omega(\lambda, v, e)(u)$ be the $\lambda$-Schubert variety in $G(d, n)_{v}(u)$ corresponding to the flag at $p(v, e)$. Continuing our abuses of notation, we will also write $\Omega(\lambda, v, e)(u)$ for the preimage of this subvariety in any product $\prod_{i} G(d, n)_{v_{i}}(u)$ where $v_{1}=v$.

We restate Proposition 3.1 in the language we have developed.

Proposition 3.5. Let $p_{1}, p_{2}, q_{1}$ and $q_{2} \in[r]$ with $v_{i}=v\left(p_{1}, p_{2}, q_{i}\right)$.

If $\phi\left(v_{1}\right)=\phi\left(v_{2}\right)$ then $\mathcal{X}\left(p_{1}, p_{2} ; q_{1}, q_{2}\right)(u)$ is the graph of an isomorphism between $\mathcal{G}(d, n)_{v_{1}}(u)$ and $\mathcal{G}(d, n)_{v_{2}}(u)$.

If $\phi\left(v_{1}\right) \neq \phi\left(v_{2}\right)$, let $e_{i}$ be the edge incident to $v_{i}$ which separates $v_{i}$ from $v_{3-i}$. Then $\mathcal{X}\left(p_{1}, p_{2} ; q_{1}, q_{2}\right)(u)=\bigcup_{\nu \in \Lambda} \Omega\left(\nu, v_{1}, e_{1}\right)(u) \times \Omega\left(\nu^{C}, v_{2}, e_{2}\right)(u)$.

We have the following corollaries:

Corollary 3.6. Let $\left(p_{1}, p_{2}, q_{1}, q_{2}\right)$ and $\left(p_{1}^{\prime}, p_{2}^{\prime}, q_{1}^{\prime}, q_{2}^{\prime}\right)$ be two quadruples of distinct elements of $[r]$ such that $v\left(p_{1}, p_{2}, q_{1}\right)=v\left(p_{1}^{\prime}, p_{2}^{\prime}, q_{1}^{\prime}\right)$ and $v\left(p_{1}, p_{2}, q_{2}\right)=v\left(p_{1}^{\prime}, p_{2}^{\prime}, q_{2}^{\prime}\right)$. Define these two vertices to be $v_{1}$ and $v_{2}$. Then $\mathcal{X}\left(p_{1}, p_{2} ; q_{1}, q_{2}\right)$ and $\mathcal{X}\left(p_{1}^{\prime}, p_{2}^{\prime} ; q_{1}^{\prime}, q_{2}^{\prime}\right)$ meet $G(d, n)_{v_{1}} \times_{U(\Gamma)} G(d, n)_{v_{2}}$ in the same subvariety.

Also, $\mathcal{X}\left(p_{1}, p_{2} ; q_{1}, q_{2}\right)$ and $\mathcal{X}\left(p_{1}, p_{2} ; q_{2}, q_{1}\right)$ meet $G(d, n)_{v_{1}} \times_{U(\Gamma)} G(d, n)_{v_{2}}$ in the same subvariety. 


\section{SCHUBERT PROBlems FROM STABLE CURVES}

Proof. By Proposition 3.1, all the schemes $\mathcal{X}(, ;$, ) above are reduced, so we can check the equality on point sets. So it is enough to show that, for every $u \in U(\Gamma)$, the fibers $\mathcal{X}\left(p_{1}, p_{2} ; q_{1}, q_{2}\right)(u)$ and $\mathcal{X}\left(p_{1}^{\prime}, p_{2}^{\prime} ; q_{1}^{\prime}, q_{2}^{\prime}\right)(u)$ are equal. This is immediate from the description in Proposition 3.5. The same argument shows that $\mathcal{X}\left(p_{1}, p_{2} ; q_{1}, q_{2}\right)=\mathcal{X}\left(p_{1}, p_{2} ; q_{2}, q_{1}\right)$.

So the following definition makes sense: For an unordered pair $\left\{v_{1}, v_{2}\right\} \in \Gamma^{\circ}$, we write $\mathcal{X}\left(v_{1} ; v_{2}\right)$ for the subset of $G(d, n)_{v_{1}} \times_{U(\Gamma)} G(d, n)_{v_{2}}$ cut out by $\mathcal{X}\left(p_{1}, p_{2} ; q_{1}, q_{2}\right)$ for any $\left(p_{1}, p_{2}, q_{1}, q_{2}\right)$ with $v\left(p_{1}, p_{2}, q_{1}\right)=v_{1}$ and $v\left(p_{1}, p_{2}, q_{2}\right)=v_{2}$. For an edge $e$ connecting two internal vertices $v_{1}$ and $v_{2}$ of $\Gamma$, we write $\mathcal{X}(e)$ for $\mathcal{X}\left(v_{1} ; v_{2}\right)$.

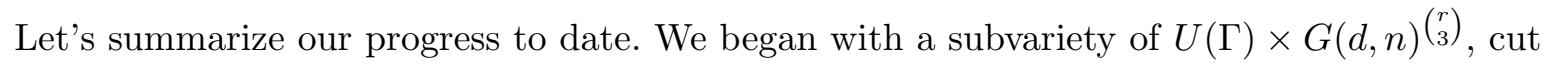
out by $r(r-1)(r-2)(r-3)$ relations. However, thanks to Proposition 3.4, we can think of this as a subvariety of $U(\Gamma) \times G(d, n)^{r-2}$, where each factor $G(d, n)$ corresponds to an internal vertex of $\Gamma$. Proposition 3.6 has reduced us to $\left(\begin{array}{c}r-2 \\ 2\end{array}\right)$ relations: one for each pair of internal vertices of $\Gamma$. Our eventual goal will be to reduce ourselves to $r-3$ relations: one for each internal edge of $\Gamma$.

For $w \in \Delta^{\circ}$ and $u \in U(\Gamma)$, let $G(d, n)_{w}(u)$ be $\bigcap_{\phi\left(v_{1}\right)=\phi\left(v_{2}\right)=w} \mathcal{X}\left(v_{1} ; v_{2}\right)(u)$, an intersection inside $\prod_{\phi(v)=w} G(d, n)_{v}(u)$.

COROLlary 3.7. With the above notation, for any $v \in \Gamma^{\circ}$ with $\phi(v)=w$, we have the isomorphism $G(d, n)_{v}(u) \cong G(d, n)_{w}(u)$.

Proof. As in the proof of Proposition 3.4, we just need to note that all of the $\mathcal{X}\left(v_{1} ; v_{2}\right)(u)$ are compatible isomorphisms.

Corollary 3.8. Let $u \in U(\Gamma)$. Let $w_{1}$ and $w_{2}$ be distinct vertices of $\Delta^{\circ}$. Let $v_{1}, v_{1}^{\prime}, v_{2}$ and $v_{2}^{\prime}$ be vertices in $\Gamma^{\circ}$ with $\phi\left(v_{i}\right)=\phi\left(v_{i}^{\prime}\right)=w_{i}$. Then the identifications $G(d, n)_{v_{i}}(u) \cong G(d, n)_{v_{i}^{\prime}}(u)$ for $i=1,2$ identify $\mathcal{X}\left(v_{1} ; v_{2}\right)(u)$ with $\mathcal{X}\left(v_{1}^{\prime} ; v_{2}^{\prime}\right)(u)$.

The above corollaries let us make definitions for $\Delta$ similar to those we have for $\Gamma$ : We write $\mathcal{X}\left(w_{1} ; w_{2}\right)(u)$ for $\mathcal{X}\left(v_{1} ; v_{2}\right)(u)$ with $v_{1}$ and $v_{2}$ as in the Corollary 3.8. For an edge $e$ connecting two internal vertices $w_{1}$ and $w_{2}$ of $\Delta$, we write $\mathcal{X}(e)(u)$ for $\mathcal{X}\left(w_{1} ; w_{2}\right)(u)$.

Proposition 3.9. The intersection $\bigcap_{e} \mathcal{X}(e)$, where e runs over the $r-3$ internal edges of $\Gamma$, is Cohen-Macaulay, reduced and flat of relative dimension $d(n-d)$ over $U(\Gamma)$.

Proof. By Proposition 3.4, $G(d, n)_{v}$ is a trivial bundle over $U(\Gamma)$ with fibers isomorphic to $G(d, n)$. So $\prod_{v \in \Gamma^{\circ}} G(d, n)_{v}$ is a trivial bundle with fibers $G(d, n)^{r-2}$. In particular, the fibers are smooth of dimension $(r-2) d(n-d)$. And each $\mathcal{X}(e)$ is Cohen-Macaulay of codimension $d(n-d)$ (Proposition 3.1). In the next several paragraphs, our goal is to show that the fibers of $\bigcap_{e} \mathcal{X}(e)$ are of the expected dimension: namely, $(r-2) d(n-d)-(r-3) d(n-d)=d(n-d)$.

Let $u \in U(\Gamma)$. We use the notations $\Delta$, $\phi$, etc., that we have associated with $u$ above. Let $s=\left|\Delta^{\circ}\right|$.

First consider the intersection $\bigcap_{\phi}$ collapses $e^{\mathcal{X}(e)}$, in the fiber over $u$, where we only intersect over edges $e$ of $\Gamma$ collapsed by $\phi$. By Corollaries 3.7 and 3.8 , this intersection is $G(d, n)^{s}$ and, for $e$ an internal edge of $\Delta$, it makes sense to talk about the subvariety $\mathcal{X}(e)$ in this $G(d, n)^{s}$. So, instead of studying the subvariety $\bigcap_{e \in \Gamma_{1}^{\circ}} \mathcal{X}(e)$ of $G(d, n)^{r-2}$, we can study the subvariety $\bigcap_{e \in \Delta_{1}^{\circ}} \mathcal{X}(e)$ of $G(d, n)^{s}$.

Let $w \in \Delta^{\circ}$. Consider the intersection $\bigcap_{e \in \Delta_{1}^{\circ}} \mathcal{X}(e)(u)$. Let $e$ be an internal edge of $\Delta$ connecting $v_{1}$ and $v_{2}$. By Proposition 3.5,

$$
\mathcal{X}(e)(u)=\bigcup_{\nu \in \Lambda} \Omega\left(\nu, v_{1}, e\right)(u) \times \Omega\left(\nu^{C}, v_{2}, e\right)(u) .
$$




\section{DAVID E SPEYER}

Let $N$ be the set of maps $\nu$ which, to each pair $(v, e)$ of edge $e \in \Delta_{1}^{\circ}$ and endpoint $v$ of $e$, assigns a partition $\nu(v, e)$ obeying the following condition: For $v_{1}$ and $v_{2}$ the two endpoints of $e$, we have $\nu\left(v_{1}, e\right)=\nu\left(v_{2}, e\right)^{C}$.

Since we are working on the level of pointsets, intersection distributes over union and we deduce that

$$
\bigcap_{e \in \Delta_{1}^{\circ}} \mathcal{X}(e)(u)=\bigcup_{\nu \in N} \bigcap_{e \in \Delta_{1}^{\circ}} \Omega\left(\nu\left(v_{1}, e\right), v_{1}, e\right)(u) \times \Omega\left(\nu\left(v_{2}, e\right), v_{2}, e\right)(u),
$$

where $v_{1}$ and $v_{2}$ are the endpoints of $e$. We can regroup the right-hand side as

$$
\bigcup_{\nu \in N} \prod_{v \in \Delta^{\circ}} \bigcap_{\substack{e \ni v \\ e \in \Delta_{1}^{\circ}}} \Omega(\nu(v, e), v, e)(u)
$$

We have engaged in an abuse of notation, where the $\Omega(\nu(v, e), v, e)(u)$ in the two displayed equations denote subvarieties of products $\prod G(d, n)_{v_{i}}(u)$ with different sequences $v_{i}$.

We will show that every nonempty term in the left-hand union has dimension $d(n-d)$, as desired. By Proposition 2.2, the intersection $\bigcap_{e \ni v} \Omega(\nu(v, e), v, e)(u)$ has codimension $\sum_{e \ni v}|\nu(v, e)|$. So the dimension of the $\nu$-term is

$$
s d(n-d)-\sum_{v \in \Delta^{\circ}} \sum_{\substack{e \ni v \\ e \in \Delta_{1}^{\circ}}}|\nu(v, e)| .
$$

For each edge $e \in \Delta_{1}^{\circ}$, group together the two terms coming from $e$; their sum is $d(n-d)$. So the above expression is

$$
s d(n-d)-\left|\Delta_{1}^{\circ}\right| d(n-d)=s d(n-d)-(s-1) d(n-d)=d(n-d),
$$

as desired.

We have now shown that the fibers of $\bigcap_{e} \mathcal{X}(e)$ are of the expected dimension. By Corollary 2.6, this shows that $\bigcap_{e} \mathcal{X}(e)$ is Cohen-Macaulay and is flat over $U(\Gamma)$. We still must show that $\bigcap_{e} \mathcal{X}(e)$ is reduced. Cohen-Macaulay varieties, if generically reduced, are reduced, so we just need to show that $\bigcap_{e} \mathcal{X}(e)$ is generically reduced. So it is enough to check that, for $u \in U(\Gamma) \cap M_{0, r}$, the fiber $\bigcap_{e} \mathcal{X}(e)(u)$ is reduced. This fiber is isomorphic to $G(d, n)$.

Proposition 3.10. The intersection $\bigcap_{e} \mathcal{X}(e)$, inside $\prod_{v \in \Gamma^{\circ}} G(d, n)_{v}$, is $\mathcal{G}(d, n)$.

Proof. As discussed above, we have $\mathcal{G}(d, n)=\bigcap \mathcal{X}\left(v ; v^{\prime}\right)$, where $\left\{v, v^{\prime}\right\}$ runs over all pairs of internal vertices of $\Gamma$. We want to show that $\bigcap_{v, v^{\prime} \in \Gamma^{\circ}} \mathcal{X}\left(v ; v^{\prime}\right)=\bigcap_{\Gamma_{1}^{\circ}} \mathcal{X}(e)$. The right-hand side is the intersection of a smaller collection of varieties than the left-hand side, so we have $\bigcap \mathcal{X}\left(v ; v^{\prime}\right) \subseteq \bigcap \mathcal{X}(e)$.

To prove the reverse containment, we must establish that, for any two internal vertices $v$ and $v^{\prime}$ of $\Gamma$, we have $\mathcal{X}\left(v ; v^{\prime}\right) \supseteq \bigcap \mathcal{X}(e)$. We showed above that $\bigcap \mathcal{X}(e)$ is reduced, so it is enough to check this containment on point sets. Even better, we showed above that $\bigcap \mathcal{X}(e)$ is flat over $U(\Gamma)$. So it is enough to check this containment over an open subset of $U(\Gamma)$.

We work over the open subset $U_{0}=U(\Gamma) \cap M_{0, r}$. Let $v=v_{0}, v_{1}, v_{2}, \ldots, v^{\prime}=v_{\ell}$ be the path through $\Delta$ from $v$ to $v^{\prime}$. Over these open subsets, all of the families $\mathcal{X}\left(v_{i-1} ; v_{i}\right)$ are graphs of isomorphisms and the projection of $\bigcap \mathcal{X}\left(v_{i-1} ; v_{i}\right)$ onto $G(d, n)_{v} \times_{U_{0}} G(d, n)_{v^{\prime}}$ is the composite isomorphism. Moreover, this composite isomorphism is $\mathcal{X}\left(v ; v^{\prime}\right)$. So $\mathcal{X}\left(v ; v^{\prime}\right) \supseteq \bigcap_{i=1}^{r} \mathcal{X}\left(v_{i-1} ; v_{i}\right)$ over $U_{0}$, as desired.

We are now ready to prove the first of the main theorems: 


\section{SCHUBERT PROBlEMS FROM STABLE CURVES}

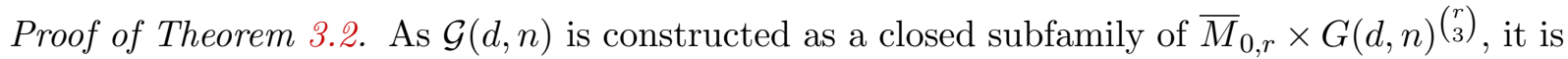
projective over $\bar{M}_{0, r}$. Fix a trivalent tree $\Gamma$. Over $U(\Gamma)$, we have shown that $\bigcap_{e \in \Gamma} \mathcal{X}(e)=\mathcal{G}(d, n)$, and that the left-hand intersection is Cohen-Macaulay, reduced, and flat over $U(\Gamma)$. Since the conditions of being Cohen-Macaulay, reduced, and flat are local on the base, and since the $U(\Gamma)$ cover $\bar{M}_{0, r}$, this shows that $\mathcal{G}(d, n)$ is Cohen-Macaulay, reduced, and flat over $\bar{M}_{0, r}$.

We now prove Theorem 1.1. Fix partitions $\lambda_{1}, \lambda_{2}, \ldots, \lambda_{r}$ in $\Lambda$. Let $\Gamma$ be as before.

Proposition 3.11. Let $t \in[r]$ and let $v \in \Gamma^{\circ}$ be the vertex adjacent to $t$. Let $p, q$ and $p^{\prime}, q^{\prime}$ be in $[r]$ so that $v(p, q, t)=v\left(p^{\prime}, q^{\prime}, t\right)=v$. Then $\Omega\left(\lambda_{t}, t\right)_{p, q, t} \cap G(d, n)_{v}=\Omega\left(\lambda_{t}, t\right)_{p^{\prime}, q^{\prime}, t} \cap G(d, n)_{v}$ over $U(\Gamma)$.

Proof. We may exchange labels so that $p$ and $p^{\prime}$ are in one component of $\Gamma \backslash v$ and $q$ and $q^{\prime}$ are in the other. The isomorphism $\mathcal{X}\left(p, t ; q, q^{\prime}\right)$ equates $\Omega\left(\lambda_{t}, t\right)_{p, q, t}$ and $\Omega\left(\lambda_{t}, t\right)_{p, q^{\prime}, t}$; the isomorphism $\mathcal{X}\left(q, t ; p, p^{\prime}\right)$ equates $\Omega\left(\lambda_{t}, t\right)_{p, q^{\prime}, t}$ and $\Omega\left(\lambda_{t}, t\right)_{p^{\prime}, q^{\prime}, t}$.

So it makes sense to talk about the Schubert variety $\Omega\left(\lambda_{t}, t\right)$ in $G(d, n)_{v}$ without talking about which $T$ we are using to define it. And $\mathcal{S}\left(\lambda_{\bullet}\right)$ over $U(\Gamma)$ is $\mathcal{G}(d, n) \cap \bigcap_{t \in[r]} \Omega\left(\lambda_{t}, t\right)$. Since Schubert varieties are Cohen-Macaulay ([Ram85]), $\Omega\left(\lambda_{t}, t\right)$ is Cohen-Macaulay. Let $u \in U(\Gamma)$. Reuse the notations $\Delta$ and so forth. We write $\Delta_{1}$ for the edge set of $\Delta$.

Proofs of Theorems 1.1 and 1.2. As in the proof of Theorem 3.2, it is enough to check the claim over $U(\Gamma)$; all of the statements which follow take place over $U(\Gamma)$ (or specified subsets thereof). The family $\prod_{v \in \Gamma^{\circ}} G(d, n)_{v}$ has dimension $(r-2) d(n-d)$ over $U(\Gamma)$. The Cohen-Macaulay family $\mathcal{G}(d, n)$ has codimension $(r-3) d(n-d)$; the Cohen-Macaulay family $\bigcap_{t \in[r]} \Omega\left(\lambda_{t}, t\right)$ has codimension $\sum_{t \in[r]}\left|\lambda_{t}\right|$. So, to prove that the intersection is Cohen-Macaulay and flat, we simply must check that all the fibers have the expected dimension.

Fix $u \in U(\Gamma)$; we will compute the dimension of the fibers over $u$. As in the proof of Theorem 3.2 , this fiber is

$$
\bigcup_{\nu \in N} \prod_{v \in \Delta^{\circ}}\left(\bigcap_{\substack{e \ni v \\ e \in \Delta_{1}^{\circ}}} \Omega(\nu(v, e), v, e)(u) \cap \bigcap_{\substack{t \in[r] \\ t \text { neighbors } v}} \Omega\left(\lambda_{t}, t\right)_{v}\right) .
$$

As in the proof of Proposition 3.9, this has dimension

$$
s d(n-d)-\sum_{v \in \Delta^{\circ}} \sum_{\substack{e \ni v \\ e \in \Delta_{1}^{\circ}}}|\nu(v, e)|-\sum_{t \in[r]}\left|\lambda_{t}\right|=d(n-d)-\sum\left|\lambda_{t}\right| .
$$

We now deduce the Cohen-Macaulayness and flatness as before.

What remains is to check reducedness of a generic fiber; this again follows by Proposition 2.2.

In the course of our proof, we have also described the fibers of $\mathcal{S}\left(\lambda_{\bullet}\right)$ over $U(\Gamma)$. Our description is that of Theorem 1.2.

We also check Proposition 1.3.

Proof of Proposition 1.3. Let $\sum_{t \in[r]}\left|\lambda_{t}\right|=d(n-d)$. Fix a stable curve $C$. Fix a node labeling $\nu$ as in Theorem 1.2. If $\nu$ contributes a nonempty variety to the above union, then every factor 


\section{DAVID E SPEYER}

in the product over $v \in \Delta^{\circ}$ must be nonempty. Using Theorem 2.2, for every vertex $v \in \Delta^{\circ}$, we have

$$
\sum_{x \in C_{j}}\left|\nu\left(x, C_{j}\right)\right|=\sum_{\substack{e \ni v \\ e \in \Delta_{1}^{\circ}}}|\nu(v, e)|+\sum_{\substack{t \in[r] \\ t \text { neighbors } v}}\left|\lambda_{t}\right| \leqslant d(n-d) .
$$

Summing over all $v$ as in the proof of Proposition 3.9, the above inequality implies that $\sum_{t \in[r]}\left|\lambda_{t}\right| \leqslant d(n-d)$. But our hypothesis is that $\sum\left|\lambda_{t}\right|=d(n-d)$, so the above inequality must be an equality for every $v$.

Finally, we move on to the claim about real points.

Proof of Theorem 1.4. Let $\sum_{t \in[r]}\left|\lambda_{t}\right|=d(n-d)$. Let $u \in \bar{M}_{0, r}(\mathbb{R})$. We must consider the fiber of $\mathcal{S}\left(\lambda_{\bullet}\right)$ over $u$. This fiber is

$$
\bigcup_{\nu \in N} \prod_{v \in \Delta^{\circ}}\left(\bigcap_{\substack{e \ni v \\ e \in \Delta_{1}^{\circ}}} \Omega(\nu(v, e), v, e)(u) \cap \bigcap_{\substack{t \in[r] \\ t \text { neighbors } v}} \Omega\left(\lambda_{t}, t\right)_{v}\right) .
$$

By Proposition 1.3, we only get a nonempty contribution to the union when

$$
\sum_{x \in C_{j}}\left|\nu\left(C_{j}, x\right)\right|=d(n-d)
$$

for every $v \in \Delta^{\circ}$. By the MTV Theorem (Theorem 2.3), the term in the large parenthesis is a reduced union of real points. So the interior fiber of $\mathcal{S}\left(\lambda_{\bullet}\right)$ over $u$ is a reduced union of real points, as desired.

For the rest of the paper, we will be interested in the case where $\sum_{t \in[r]}\left|\lambda_{t}\right|=d(n-d)$, and will be primarily interested in the case of real points. Let's emphasize what our results say in this case. The family $\mathcal{S}\left(\lambda_{\bullet}\right)$ is a finite cover of $\bar{M}_{0, r}(\mathbb{R})$. For $u \in \bar{M}_{0, r}(\mathbb{R})$, let $\Delta$ be the tree for $u$ as before. There are finitely many points of $\mathcal{S}\left(\lambda_{\bullet}\right)$ above $u$; fix one such point $x$. For every vertex $v \in \Delta^{\circ}$, and edge $e \in \Delta_{1}$ incident to $e$, the point $x$ assigns a partition $\nu(e, v)$ to $(v, e)$. When $e \in \Delta_{1}^{\circ}$, we have $\nu(v, e)=\nu(v, e)^{C}$; when $e$ joins $v$ to the leaf $t$, we have $\nu(e, v)=\lambda_{t}$. At every vertex $v \in \Delta^{\circ}$, we have $\sum_{e \ni v}|\nu(e, v)|=d(n-d)$.

Remark: It is straightforward to make analogous definitions for general $G / P$. However, Proposition 2.2 fails [RSSS06, Section 3.3.6], which makes it hard to make nontrivial statements about these definitions.

\subsection{Relation to the work of Eisenbud and Harris}

Eisenbud and Harris, [EH87], introduce a similar and more general construction, which deals with any nodal curve whose dual graph is a tree, not only a stable curve of genus 0 . Consider [EH87, Theorem 3.3] with (in the notation of that theorem) $\pi: X \rightarrow B$ being the universal curve $\mathcal{C}$ over $\bar{M}_{0, n}$, with $p_{1}, \ldots, p_{s}$ the marked points and with $b_{1}, \ldots, b_{s}$ corresponding to the partitions $\lambda_{i}$ by a simple combinatorial rule which we omit. That theorem then constructs ${ }^{1}$

\footnotetext{
${ }^{1}$ One of the hypotheses of that theorem is that there is a relatively ample divisor $D$ on $X$ with $D \cap \bigcup_{i} p_{i}(B)=\emptyset$. This condition holds for $\pi: \mathcal{C} \rightarrow \bar{M}_{0, n}$. Kapranov [Kap93] embeds $\mathcal{C}$ into $\bar{M}_{0, n} \times \mathbb{P}^{n-2}$ so that the marked points land at $n$ fixed points of $\mathbb{P}^{n-2}$, independent of the choice of curve. Take any hyperplane $H \subset \mathbb{P}^{n-2}$ not passing through those $n-2$ points and pull it back to $\mathcal{C}$ to get a relatively ample divisor of the required form.
} 


\section{SCHUBERT PROBlEMS FROM STABLE CURVES}

a family $G_{d}^{r}\left(X / B,\left(p_{1}, b_{1}\right), \ldots,\left(p_{s}, b_{s}\right)\right) \rightarrow \bar{M}_{0, n}$; I'll abbreviate this family to $G_{d}^{r}$. What is the relation between $G_{d}^{r}$ and $\mathcal{S}\left(\lambda_{\bullet}\right)$ ?

If the ramification conditions $b_{1}, \ldots, b_{s}$ are empty, then I believe (but have not proved) that Eisenbud and Harris's $G_{d}^{r}$ is our $\mathcal{G}(r+1, r+d+1)$. For other ramification conditions, I believe we obtain an open subvariety of $\mathcal{S}\left(\lambda_{\bullet}\right)$; it is an open subvariety because Eisenbud and Harris work with the analogues of Schubert cells, not Schubert varieties. Because they work with an open subvariety, their family is only stated to be quasi-projective, not projective. Also, Eisenbud and Harris do not discuss flatness or the Cohen-Macaulay property. There are related, but not equivalent, results in their Corollaries 3.5 and 3.7. Part of why they do not prove these results is that the obvious generalizations are not true in higher genus; see their Example 3.2. Finally, Eisenbud and Harris's proofs do not provide the sort of explicit coordinates that ours do, which will be important for computations.

\section{Monodromy along Curves}

Recall that we write $\square$ for the partition (1). Our goal in this section is to understand the family $\mathcal{S}(\lambda, \mu, \square, \square)$, where $|\lambda|+|\mu|=d(n-d)-2$. So $\mathcal{S}(\lambda, \mu, \square, \square)$ is a finite cover of $\bar{M}_{0,4} \cong \mathbb{P}^{1}$.

We first recall (a special case of) Pieri's rule [Sta99, Theorem 7.15.7]:

Proposition 4.1. Let $|\lambda|+|\mu|=d(n-d)-1$. The Littlewood-Richardson coefficient $c_{\lambda \mu \square}^{m}$ is 1 if $\mu^{C}$ is obtained by adding a single box to $\lambda$, and 0 otherwise.

Corollary 4.2. Let $|\lambda|+|\mu|=d(n-d)-2$. The Littlewood-Richardson coefficient $c_{\lambda \mu \square \square}^{\boxplus m}$ is 2 if $\mu^{C}$ is obtained by adding two nonadjacent boxes to $\lambda$; it is 1 if $\mu^{C}$ is obtained by adding a horizontal or vertical domino to $\lambda$, and is 0 otherwise.

Proof. We want to compute $c_{\lambda \square \square}^{\mu^{C}}$. By the associativity of the Littlewood-Richardson product, we can write

$$
c_{\lambda \square \square}^{\mu^{C}}=\sum_{\kappa} c_{\lambda \square}^{\kappa} c_{\kappa \square}^{\mu^{C}} .
$$

We can evaluate each term on the right-hand side using Proposition 4.1. The only nonzero terms are where $\kappa$ is obtained by adding a single box to $\lambda$, and $\mu^{C}$ is obtained by adding a single box to $\kappa$. So the sum is zero unless $\mu^{C}$ is obtained by adding two boxes to $\lambda$. If those two boxes are adjacent, there is one possible intermediate $\kappa$; if the two boxes are not adjacent, there are two possible terms.

Corollary 4.3. If $\mu^{C}$ does not contain $\lambda$, then $\mathcal{S}(\lambda, \mu, \square, \square)$ is empty. If $\mu^{C} \backslash \lambda$ is a horizontal or vertical domino, then $\mathcal{S}(\lambda, \mu, \square, \square) \cong \mathbb{P}^{1}$, and the map to $\bar{M}_{0,4}$ is an isomorphism.

Proof. The first sentence is immediate. The second is because a flat projective degree 1 map to a normal variety must be an isomorphism.

From now on, we assume that we are in the remaining case, where $\mu^{C} \backslash \lambda$ is two nonadjacent squares. Let $\kappa_{1}$ and $\kappa_{2}$ be the two partitions lying between $\lambda$ and $\mu^{C}$, with the added box of $\kappa_{1}$ to the southwest of the added box of $\kappa_{2}$. For example, we might have $\lambda=(3,1)$ and $\mu^{C}=(4,2)$, in which case $\kappa_{1}=(3,2)$ and $\kappa_{2}=(4,1)$. The next theorem summarizes our results.

ThEOREM 4.4. The family $\mathcal{S}(\lambda, \mu, \square, \square)$ is a curve of genus 0 . The degree 2 map from $\mathcal{S}(\lambda, \mu, \square, \square)$ to $\bar{M}_{0,4}$ is branched over two non-real points. 


\section{DAVID E SPEYER}

As we travel around the circle $\mathcal{S}(\lambda, \mu, \square, \square)(\mathbb{R})$, we encircle $\bar{M}_{0,4}$ twice, and thus the six points which lie over the boundary of $\bar{M}_{0,4}$ acquire a circular ordering. Each of these orderings corresponds to a stable curve with one node and that node is labeled by a pair of partitions $\left(\nu, \nu^{C}\right)$ as discussed in the previous section. The circular ordering of the $\nu$ 's is as follows:
(1) $\left(\kappa_{1}, \kappa_{1}^{C}\right)$
(4) $\left(\kappa_{2}, \kappa_{2}^{C}\right)$
(2) $\left(\kappa_{1}, \kappa_{1}^{C}\right)$
(3) $\left((1,1),(1,1)^{C}\right)$
(5) $\left(\kappa_{1}, \kappa_{1}^{C}\right)$
(6) $\left((2),(2)^{C}\right)$.

This result should be compared with Purbhoo's [Pur13, Theorem 2.3].

REMARK 4.5. We do not actually use the knowledge of which of points (3) and (6) is which. At the end of Section 7, we will be able to give a more conceptual proof that points (3) and (6) are positioned as claimed. However, determining this is a straightforward though messy computation, given the other computations in this section, and it seemed silly not to include the answer. Because we do not need the answer, we omit the final steps of the computation.

Lemma 4.6. Consider the conic $K$ given by $a u^{2}-b u-c \tau u+d \tau=0$ with positive real coefficients $a, b, c, d$. If $b c>a d$, then the projective completion of $K(\mathbb{R})$ projects to the real $\tau$-line in an unbranched 2 to 1 cover.

Proof. We complete the $(\tau, u)$ plane to $\mathbb{P}^{2}$, with coordinates $(\tau: u: 1)$. The given conic corresponds to the symmetric form with matrix

$$
\left(\begin{array}{ccc}
0 & -c / 2 & d / 2 \\
-c / 2 & a & -b / 2 \\
d / 2 & -b / 2 & 0
\end{array}\right) .
$$

Since the determinant of this matrix is $-d / 4(a d-b c)>0$, and it has a 0 on the diagonal, it must have signature +--. Thus, the interior of $K(\mathbb{R})$ is the region where the quadratic is positive. In particular, the point with homogeneous coordinates $(0: 1: 0)$ is within this interior. Projection onto the $\tau$-line is projection from this point, so the mapping is unbranched.

Figure 2 shows the conic $u^{2}-2 u-2 \tau u+3 \tau=0$, which we discuss in Example 4.8. (The asymptotes and marked points of the figure will be explained as they become relevant.) The map to the $\tau$-line is the vertical projection.

Recall the notations $I(\lambda)$ and $\Delta(S)$ from Proposition 2.1 and the paragraphs preceding it. Let $L=I(\lambda)$ and $M=I\left(\mu^{C}\right)$. Then $L$ and $M$ are of the form $S f g$ and $S(f+1)(g+1)$, respectively, for some $S \in\left(\begin{array}{c}{[n]} \\ d-2\end{array}\right)$ and some $f, g$; we have $f<f+1<g<g+1$ and all four of these indices are in $[n] \backslash S$. We have $I\left(\kappa_{1}\right)=S(f+1) g$ and $I\left(\kappa_{2}\right)=S f(g+1)$. Abbreviate these to $K_{1}$ and $K_{2}$.

Lemma 4.7. Let $\tau \in \mathbb{C P}^{1} \backslash\{0,1, \infty\}$. Consider the Schubert problem $(\lambda, \mu, \square, \square)$, with respect to the osculating flags at $(0, \infty, 1, \tau)$. The only nonzero solutions of the Plücker coordinates are $p_{L}, p_{K_{1}}, p_{K_{2}}$ and $p_{M}$. Those values are

$$
\begin{array}{rc}
p_{L}= & \Delta(L)^{-1} \cdot 1, \\
p_{K_{1}}= & \frac{g-f-1}{g-f} \Delta\left(K_{1}\right)^{-1} \cdot u, \\
p_{K_{2}}= & \frac{g-f+1}{g-f} \Delta\left(K_{2}\right)^{-1} \cdot\left(\tau u^{-1}\right), \\
p_{M}= & \Delta(M)^{-1} \cdot \tau,
\end{array}
$$

where $(\tau, u)$ lies on the conic

$$
(g-f-1) u^{2}-(g-f) u-(g-f) \tau u+(g-f+1) \tau=0 .
$$




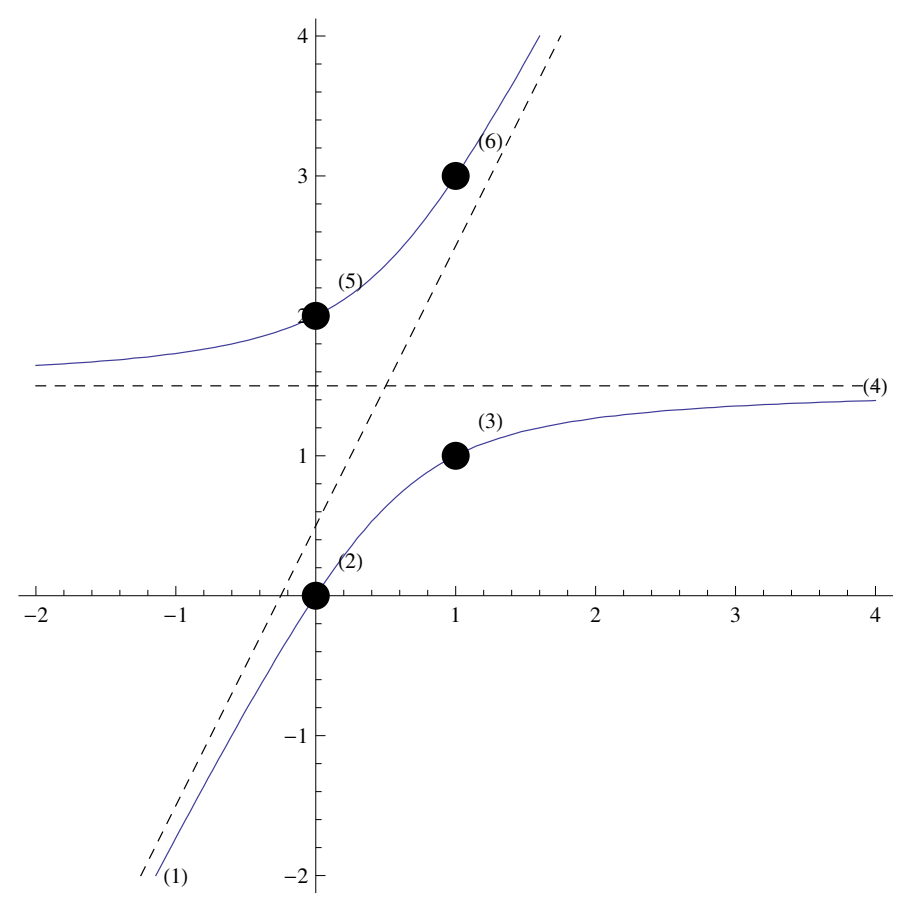

Figure 2. The conic $u^{2}-2 u-2 \tau u+3 \tau=0$ with its asymptotes and the 6 points of Theorem 4.4

EXAmple 4.8. Consider the Schubert condition $\mathcal{S}(\square, \square, \square, \square)$ in $G(2,4)$. Its solutions are given by

$$
\left(p_{12}, p_{13}, p_{23}, p_{14}, p_{24}, p_{34}\right)=(0, \tau / 2, u / 2, \tau /(2 u), 1 / 2,0)
$$

where $\tau$ and $u$ obey

$$
u^{2}-2 u-2 \tau u+3 \tau=0 .
$$

Observe that, considered as a quadratic in $u$, the discriminant of this equation is $4\left(\tau^{2}-\tau+1\right)$, so there are two distinct roots of $u$ at all values of $\tau$ other than $(1 \pm \sqrt{-3}) / 2$.

Proof. From the Plücker conditions at 0 and $\infty$, we see that the only nonzero Plücker coordinates are $p_{L}, p_{K_{1}}, p_{K_{2}}$ and $p_{M}$. They obey the relation

$$
p_{L} p_{M}=p_{K_{1}} p_{K_{2}},
$$

because there is a three-term Plücker relation whose third term is $p_{S f(f+1)} p_{S g(g+1)}$, and the variables in this last term are zero.

By Proposition 2.1, the quadratic

$$
p_{L} \Delta(L)-\left(p_{K_{1}} \Delta\left(K_{1}\right)+p_{K_{2}} \Delta\left(K_{2}\right)\right) z+p_{M} \Delta(M) z^{2}
$$

has roots at 1 and $\tau$. By rescaling the Plücker coordinates, we may assume that

$$
\left(p_{L} \Delta(L), p_{K_{1}} \Delta\left(K_{1}\right)+p_{K_{2}} \Delta\left(K_{2}\right), p_{M} \Delta(M)\right)=(\tau, 1+\tau, 1) .
$$

Plugging this into equation $(*)$, we have

$$
p_{K_{1}} p_{K_{2}}=\frac{\tau}{\Delta(L) \Delta(M)} .
$$




\section{DAVID E SPEYER}

We introduce the notation $\Delta(S, a)$ for $\prod_{s \in S}|a-s|$. So

$$
\begin{array}{rcc}
\Delta(L)= & (g-f) \cdot \Delta(S) \cdot \Delta(S, f) & \cdot \Delta(S, g), \\
\Delta\left(K_{1}\right)= & (g-f-1) \cdot \Delta(S) \cdot \Delta(S, f+1) \cdot \Delta(S, g), \\
\Delta\left(K_{2}\right)= & (g-f+1) \cdot \Delta(S) \cdot \Delta(S, f) \quad \cdot \Delta(S, g+1), \\
\Delta(M)= & (g-f) \cdot \Delta(S) \cdot \Delta(S, f+1) \cdot \Delta(S, g+1),
\end{array}
$$

and we have

$$
p_{K_{1}} p_{K_{2}}=\frac{\tau}{\Delta(S)^{2} \Delta(S, f) \Delta(S, f+1) \Delta(S, g) \Delta(S, g+1)(g-f)^{2}} .
$$

So there is some $u$ with

$$
\begin{aligned}
u & =\Delta(S) \Delta(S, f+1) \Delta(S, g)(g-f) \cdot p_{K_{1}}=\frac{g-f}{g-f-1} \Delta\left(K_{1}\right) p_{K_{1}}, \\
\tau u^{-1} & =\Delta(S) \Delta(S, f) \Delta(S, g+1)(g-f) \cdot p_{K_{2}}=\frac{g-f}{g-f+1} \Delta\left(K_{2}\right) p_{K_{2}} .
\end{aligned}
$$

We thus have

$$
p_{K_{1}} \Delta\left(K_{1}\right)+p_{K_{2}} \Delta\left(K_{2}\right)=\frac{g-f-1}{g-f} u+\frac{g-f+1}{g-f} \tau u^{-1}=1+\tau
$$

or

$$
(g-f-1) u^{2}-(g-f) u-(g-f) \tau u+(g-f+1) \tau=0 .
$$

All steps are trivially reversible.

Proof of Theorem 4.4. By Lemma 4.7 we see that, for $\tau \in M_{0,4}$, the variety $\mathcal{S}(\lambda, \mu, \square, \square)$ is parametrized by the conic

$$
(g-f-1) u^{2}-(g-f) u-(g-f) \tau u+(g-f+1) \tau=0 .
$$

We denote the projective closure of this curve by $C$. This is a hyperbola with a two asymptotes: A slant asymptote at $u=(g-1) /(g-f-1) \tau+1 /(g-f)(g-f-1)$, and a horizontal asymptote at $u=(g-f) /(g-f+1)$. As it is a conic, $C$ has genus 0 . Moreover, since $(g-f-1)(g-f+1)=$ $(g-f)^{2}-1<(g-f)^{2}$, by Lemma $4.6, C(\mathbb{R})$ is an unbranched double cover of $M_{0,4}(\mathbb{R})$.

Since $\mathcal{S}(\lambda, \mu, \square, \square)$ is closed in $\mathcal{G}(d, n)$, and $C$ is normal, the map over $M_{0,4}$ extends to a map $C \rightarrow \mathcal{S}(\lambda, \mu, \square, \square)$. Since $\mathcal{S}(\lambda, \mu, \square, \square)$ is flat over $\bar{M}_{0,4}$, the whole family $\mathcal{S}(\lambda, \mu, \square, \square)$ is the closure of the part of the family over $M_{0,4}$, and so the map $C \rightarrow \mathcal{S}(\lambda, \mu, \square, \square)$ is surjective. We now compute exactly what this map does over 0,1 and $\infty$.

The points on $C$ over $\tau=\infty$ are the endpoints of the slant asymptote and the horizontal asymptote. The points on $C$ over $\tau=0$ are $(0,0)$ and $(0,(g-f) /(g-f-1))$; the points over $\tau=1$ are $(1,1)$ and $(1,(g-f+1) /(g-f-1))$. As we travel along $C(\mathbb{R})$ in the direction of increasing $\tau$, starting at the end point of the slant asymptote, the $u$-coordinate is continually increasing, which forces these points to be ordered as follows: The end of the slant asymptote, $(0,0),(1,1)$, the end of the horizontal asymptote, $(0,(g-f) /(g-f-1)),(1,(g-f+1) /(g-f-1))$.

These points are marked in Figure 2. Points (2), (3), (5) and (6) are in the figure, and marked with solid dots; the points (1) and (4) are at the end of the asymptotes, out of the frame. 


\section{SCHUBERT PROBlems FROM STABLE CURVES}

Plugging this into Lemma 4.7, the corresponding Plücker coordinates are

$$
\begin{aligned}
& \left(p_{12}: \quad p_{13}: p_{23}: p_{14}: p_{24}: p_{34}\right)= \\
& \left(\begin{array}{lllllllll}
0: & 0 & : & * & : & 0 & * & : & 0
\end{array}\right) \text {, }
\end{aligned}
$$

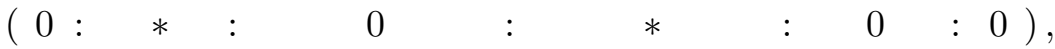

$$
\begin{aligned}
& \left(0: \Delta(L)^{-1}: \frac{g-f-1}{g-f} \Delta\left(K_{1}\right)^{-1}: \frac{g-f+1}{g-f} \Delta\left(K_{2}\right)^{-1}: \Delta(M)^{-1}: 0\right) \text {, } \\
& \left(\begin{array}{lllllllllll}
0: & 0 & : & 0 & : & * & * & : & 0
\end{array}\right) \text {, }
\end{aligned}
$$

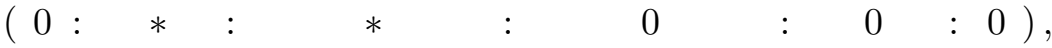

$$
\begin{aligned}
& \left(0: \Delta(L)^{-1}: \frac{g-f+1}{g-f} \Delta\left(K_{1}\right)^{-1}: \frac{g-f-1}{g-f} \Delta\left(K_{2}\right)^{-1}: \Delta(M)^{-1}: 0\right) \text { ). }
\end{aligned}
$$

Here the $*$ 's indicate nonzero numbers whose values will be irrelevant to the argument.

Note that these are the Plücker coordinates on $G(d, n)_{T}$, where $T$ indexes the subset $(0,1, \infty)$ of $(0, \infty, 1, \tau)$. In particular the projection from $\mathcal{S}(\lambda, \mu, \square, \square)$ to this Grassmannian does not identify the two points over any $\tau$, so the parametrization $C \rightarrow \mathcal{S}(\lambda, \mu, \square, \square)$ is bijective. A bit more of a computation checks that the composite $C \rightarrow G(d, n)_{T}$ is also smooth (as opposed to introducing a cusp), so $C \rightarrow \mathcal{S}(\lambda, \mu, \square, \square)$ is an isomorphism and $\mathcal{S}(\lambda, \mu, \square, \square) \rightarrow G(d, n)_{T}$ is a closed embedding.

We now must check that the ordering above corresponds to the ordering listed in Theorem 4.4. The terms where $p_{K_{1}}$ (respectively $p_{K_{2}}$ ) vanishes correspond to $\kappa_{2}$ (respectively $\kappa_{1}$ ).

We still must check that

$$
\left(0: \Delta(L)^{-1}: \frac{g-f-1}{g-f} \Delta\left(K_{1}\right)^{-1}: \frac{g-f+1}{g-f} \Delta\left(K_{2}\right)^{-1}: \Delta(M)^{-1}: 0\right)
$$

corresponds to the partition $(1,1)$, and

$$
\left(0: \Delta(L)^{-1}: \frac{g-f+1}{g-f} \Delta\left(K_{1}\right)^{-1}: \frac{g-f-1}{g-f} \Delta\left(K_{2}\right)^{-1}: \Delta(M)^{-1}: 0\right)
$$

corresponds to the partition (2). I leave this to the reader. As discussed in Remark 4.5, we will see a cleaner proof later.

\subsection{An example in $\mathcal{F} \ell(6)$}

We give an example in $\mathcal{F} \ell(6)$ to demonstrate which statements generalize to $\mathcal{F} \ell(n)$ and which do not. We assume some familiarity with the cohomology ring of $\mathcal{F} \ell(n)$. We impose the Schubert class 153264 with respect to 0 and the Schubert class 514623 with respect to $\infty$. This means that the components of the flag in $\mathcal{F} \ell(6)$ are the row spans of the top justified $k \times 6$-submatrices of a matrix of the form

$$
\left(\begin{array}{cccccc}
u_{1} & u_{2} & 0 & 0 & 0 & 0 \\
0 & 0 & 0 & 0 & v_{1} & v_{2} \\
0 & 0 & 1 & 0 & 0 & 0 \\
* & * & * & 0 & 0 & 0 \\
* & * & * & 0 & * & * \\
* & * & * & * & * & *
\end{array}\right)
$$

The intersection of these Schubert varieties is isomorphic to $\mathbb{P}^{1} \times \mathbb{P}^{1}$ with coordinates $\left(u_{1}\right.$ : $\left.u_{2}\right) \times\left(v_{1}: v_{2}\right)$.

Let $\square_{d}$ be the pull back to $H^{2}(\mathcal{F} \ell(n))$ of the class $\square$ in $G(d, n)$. We impose $\square_{2}$ at 1 and $\square_{3}$ at $\tau$. This gives

$$
\begin{array}{rllll}
4-3 u & -5 v & +4 u v & =0 \\
16 & -6 \tau u & -30 \tau v & +12 \tau^{2} u v & =0 .
\end{array}
$$




\section{DAVID E SPEYER}

Then $\tau$ and $v$ are related by

$$
(-12+6 \tau)+\left(1615 \tau-12 \tau^{2}\right) v+\left(-30 \tau+15 \tau^{2}\right) v^{2}=0
$$

and $\tau$ and $u$ are related by a similar equation, giving a birational curve.

This curve has genus 1 , and is branched over the four roots of

$$
256-960 \tau+1281 \tau^{2}-720 \tau^{3}+144 \tau^{4}=0 .
$$

These roots are at $0.678121,0.945553,1.41011$ and 1.96622 , so the analogue of the MTV Theorem fails.

\section{Real structure of $\bar{M}_{0, r}$}

We review the structure of $\bar{M}_{0, r}(\mathbb{R})$. An excellent introduction to this material is [Dev04]. Let $r \geqslant 3$.

$\bar{M}_{0, r}(\mathbb{R})$ is a connected compact manifold of dimension $r-3$. It has a natural regular $C W$ structure. The maximal faces are indexed by the $(r-1) ! / 2$ dihedral symmetry classes of ways to place the labels $[r]$ around a circle. For such a circular ordering, the points in the interior of the corresponding face correspond to configurations of points on $\mathbb{R P}^{1}$ with that circular order.

Each maximal face is isomorphic to the (simple) associahedron. We should think of faces of the associahedron as indexing partial triangulations of the $r$-gon, where the edges of the $r$-gon are labeled by the elements of $[r]$ in the corresponding circular order. Given a stable curve $C$, the nodes of $C$ correspond to the chords of the corresponding triangulation. If a chord separates the edges of the $[r]$-gon labeled by $A$ and the edges of the $[r]$-gon labeled by $[r] \backslash A$, then the corresponding node separates the points marked by $A$ and $[r] \backslash A$.

When we cross a wall of the $C W$-complex, corresponding to single chord drawn in the $r$-gon, the circular orders on the two sides of the wall differ by reversing the ordering on one side of the chord.

Figure 3 shows a portion of the $C W$-structure on $\bar{M}_{0,5}(\mathbb{R})$. The figure depicts four maximal (two-dimensional) faces, whose boundaries are dashed, surrounding a vertex. We draw the topologies of the stable curves corresponding to the four maximal faces, to one of the edges and to the central vertex.

\section{Cylindrical growth diagrams}

Let $r=d(n-d)$. By Theorem $1.4, \mathcal{S}(\square, \square, \ldots, \square)(\mathbb{R})$ is a covering of $\bar{M}_{0, r}(\mathbb{R})$, so we can pull back the regular $C W$-structure on $\bar{M}_{0, r}(\mathbb{R})$ to a regular $C W$-structure on $\mathcal{S}(\square, \square, \ldots, \square)(\mathbb{R})$. In this section we will describe the combinatorial data which we use to index the maximal cells of this $C W$-structure; in the next section we describe the cells themselves.

Define

$$
\mathbb{I}:=\left\{(i, j) \in \mathbb{Z}^{2}: i \leqslant j \leqslant i+r\right\} .
$$

Define a cylindrical growth diagram to be a map from $\mathbb{I}$ to $\Lambda(d, n)$ (the set of partitions contained in $\boxplus$ ) obeying

(1) $\gamma_{(i-1) j}$ and $\gamma_{i(j+1)}$ are obtained by adding a single box to $\gamma_{i j}$, and $\gamma_{i j}=|j-i|$.

(2) If the two boxes of the skew-shape $\gamma_{(i-1)(j+1)} / \gamma_{i j}$ are nonadjacent, then $\gamma_{i(j+1)} \neq \gamma_{(i+1) j}$. 


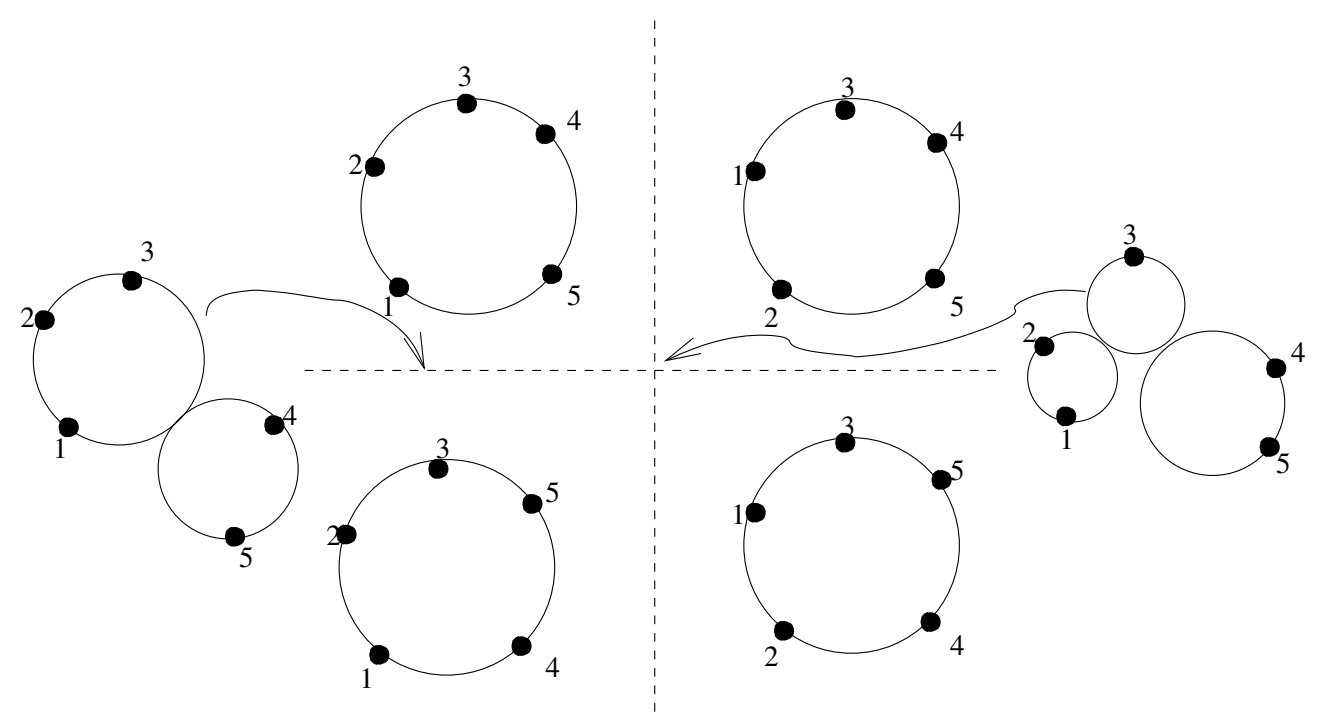

FiguRE 3. A portion of $\bar{M}_{0,5}(\mathbb{R})$

$$
\begin{aligned}
& \begin{array}{lllllll}
00 & 10 & 20 & 21 & 31 & 32 & 33
\end{array} \\
& \begin{array}{lllll}
0 & 10 & 11 & 21 & \underset{\uparrow}{22} \rightarrow 32 \rightarrow 33
\end{array} \\
& 00 \quad \underset{\uparrow}{10} \rightarrow 20 \rightarrow 21 \quad 31 \quad 32 \quad 33 \\
& \begin{array}{lllllll}
00 & 10 & 11 & 21 & 22 & 32 & 33
\end{array} \\
& \begin{array}{lllllll}
00 & 10 & 20 & 21 & 31 & 32 & 33
\end{array} \\
& \begin{array}{lllllll}
00 & 10 & 11 & 21 & 22 & 32 & 33
\end{array} \\
& \begin{array}{lllllll}
00 & 10 & 20 & 21 & 31 & 32 & 33
\end{array}
\end{aligned}
$$

FiguRE 4. A cylindrical growth diagram for $(d, n)=(2,5)$

The motivation for the term "cylindrical" will be explained after Proposition 7.4.

Figure 4 shows $r+1=7$ rows of a growth diagram with $(d, n)=(2,5)$. We omit the commas for brevity, writing 31 rather than $(3,1)$. Note that the bottom row is a repetition of the top row; this is a general phenomenon that we will discuss in Proposition 7.4.

Note that condition (1) forces the boundary conditions $\gamma_{k k}=\emptyset, \gamma_{k(k+1)}=\square, \gamma_{k(k+r-1)}=\square^{C}$ and $\gamma_{k(k+r)}=\boxplus$. Condition (2) is the growth diagram condition from [Sta99, Proposition A.1.2.7].

Define a path through $\mathbb{I}$ to be a sequence $\left(i_{0}, j_{0}\right),\left(i_{1}, j_{1}\right), \ldots,\left(i_{r}, j_{r}\right)$ of elements of $\mathbb{I}$ such that $j_{0}=i_{0}$ and, for each $0 \leqslant k<r$, the difference $\left(i_{k+1}, j_{k+1}\right)-\left(i_{k}, j_{k}\right)$ is either $(-1,0)$ or $(0,1)$. By reading a cylindrical growth diagram along a path through $\mathbb{I}$, we obtain a sequence of partitions, each of which is obtained by adding a single box to the previous one; in other words, we obtain a standard Young tableaux of shape $\boxplus$. In Figure 5, we translate the path in Figure 4 into a sequence of partitions and draw it as a Young tableaux in the standard way. 


\section{DAVID E SPEYER}

$(00,10,20,21,22,32,33)$

\begin{tabular}{|l|l|l|}
\hline 1 & 2 & 5 \\
\hline 3 & 4 & 6 \\
\hline
\end{tabular}

FIGURE 5. A sequence of partitions, and its corresponding Young tableaux, obtained from the path in Figure 4

Lemma 6.1. Let $\left(i_{0}, j_{0}\right),\left(i_{1}, j_{1}\right), \ldots,\left(i_{r}, j_{r}\right)$ be any path through $\mathbb{I}$, and fix values of $\gamma_{i_{k} j_{k}}$ in $\Lambda$ such that $\gamma_{i_{k+1} j_{k+1}}$ is obtained by adding a single box to $\gamma_{i_{k} j_{k}}$ and $\left|\gamma_{i_{k} j_{k}}\right|=j_{k}-i_{k}$. Then there is a unique way to extend $\gamma$ to a cylindrical growth diagram.

Proof. Condition (2), combined with the boundary values $\gamma_{k k}=\emptyset$ and $\gamma_{k(k+r)}=\boxplus$, gives a unique recursion.

EXAMPLE 6.2. If $\gamma_{i j}=(4,2,1), \gamma_{(i-1) j}=(4,2,2)$ and $\gamma_{(i-1)(j+1)}=(4,3,2)$, then $\gamma_{i(j+1)}$ must be $(4,3,1)$ in order to be consistent with $(2)$. If $\gamma_{i j}=(4,2,1), \gamma_{(i-1) j}=(4,3,1)$ and $\gamma_{(i-1)(j+1)}=$ $(4,4,1)$, then (2) imposes no condition, but there is only one choice for $\gamma_{(i+1) j}$ obeying (1), namely $(4,3,1)$.

\section{Cell decomposition of $\mathcal{S}\left(\lambda_{\bullet}\right)$}

Let $r=d(n-d)$ and let $\lambda_{1}=\lambda_{2}=\cdots=\lambda_{r}=\square$. In this section, we abbreviate $\mathcal{S}(\square, \square, \cdots, \square)$ to $\mathcal{S}$. Fix a circular order $(s(1), s(2), \cdots, s(d(n-d)))$ of $[r]$. This circular order gives us a maximal cell in $\bar{M}_{0, r}(\mathbb{R})$. The preimage of this face in $\mathcal{S}(\mathbb{R})$ is the disjoint union of many ${ }^{2}$ copies of the associahedron. Let's fix one copy; call it $\sigma$.

Let $[i, j]$ denote the set $(s(i), s(i+1), \ldots, s(j-1), s(j))$, wrapping around modulo $r$ if necessary. We similarly use $[i, j),(i, j]$ and $(i, j)$ to exclude one or both of the endpoints of this set.

Let $i$ and $j$ be distinct and circularly nonadjacent elements of $[r]$. Let $\sigma_{i j}$ be the facet of $\sigma$ corresponding to stable curves with two components, one containing $[i, j)$ and one containing $[j, i)$. Consider any point of $\sigma$ on $\sigma_{i j}$. There is a corresponding node of the stable curve. To each side of this node, the function $\nu$ assigns a partition. Let $g_{i j}$ be the partition assigned to the side of the node containing $[j, i)$. (We emphasize: The side away from $[i, j)$.) So we have $g_{i j}=\gamma_{j i}^{C}$.

Lemma 7.1. The partition $g_{i j}$ is independent of the choice of point on $\sigma_{i j}$.

Proof. Let $x$ and $z$ be two points on $\sigma_{i j}$. Let $X$ and $Z$ be the corresponding stable curves. Let $X_{1}$ (respectively $Z_{1}$ ) be the union of the components of $X$ (respectively $Z$ ) containing the points labeled by $[i, j)$; let $X_{2}$ and $Z_{2}$ contain the points labeled by $[j, i)$. Define $Y$ to be the stable curve defined by gluing $X_{1}$ to $Z_{2}$ at the node of each, and let $y$ be the corresponding point of $\sigma_{i j}$.

Consider any path in $\bar{M}_{0, j-i+1}(\mathbb{R})$ linking $X_{2}$ to $Z_{2}$ while maintaining the circular ordering of the points $[j, i)$. Glue the curves in this path to $X_{1}$, giving a curve in $\bar{M}_{0, r}(\mathbb{R})$. Since nothing is changing on the $X_{1}$-side of the node, the points of the $G(d, n)_{v}$ 's for the vertices $v$ on that side of the node live in the same discrete set of Schubert solutions, everywhere along the path. By continuity, these points must simply remain constant as we travel along the path. In particular, the $\nu$-label of the node is the same at both ends of the path.

So $g_{i j}$ is the same at $X$ and $Y$. Similarly, $g_{i j}$ is the same at $Y$ and $Z$.

${ }^{2}$ By the hook length formula [Sta99, Corollary 7.21.6], the degree of the cover is $r ! /\left(d^{\underline{d}}(d+1)^{\underline{d}} \cdots(n-1)^{\underline{d}}\right)$ where $x^{\underline{d}}=x(x-1)(x-2) \cdots(x-d+1)$. 


\section{Schubert PROBlems From STABle CURVES}

For $(i, j) \in \mathbb{I}$ with $2 \leqslant j-i \leqslant r-2$, set $\gamma_{i j}=g_{i j}$, where the indices on the right-hand side are reduced modulo $r$. Let $\gamma_{i i}=\emptyset, \gamma_{i(i+1)}=\square, \gamma_{i(i+r-1)}=\square^{C}$ and $\gamma_{i(i+r)}=\boxplus$.

Lemma 7.2. Given a maximal face $\sigma$ of the $C W$-structure on $\mathcal{S}(\mathbb{R})$, define $\gamma_{i j}$ by the geometric recipe above. Then $\gamma_{i j}$ is a cylindrical growth diagram.

Proof. We first check the normalization that $\left|\gamma_{i j}\right|=|j-i|$. This follows from Proposition 1.3.

We next show that $\gamma_{i(j+1)}$ is obtained by adding a single box to $\gamma_{i j}$. When $j-i=0$ or $r-1$, this is automatic, so we assume that $1 \leqslant j-i \leqslant r-2$.

Let $X$ be a stable curve with three components, one of which contains the marked point $j$ and the nodes joining those component with the other two. The other components contain the marked points $[j+1, i)$ and $[i, j)$, in circular order. Then one node is labeled by $\left(\gamma_{i j}, \gamma_{i j}^{C}\right)$ and the other is labeled by $\left(\gamma_{i(j+1)}, \gamma_{i(j+1)}^{C}\right)$. The central component has labels $\gamma_{i j}, \gamma_{i(j+1)}^{C}$ and $\square$. So

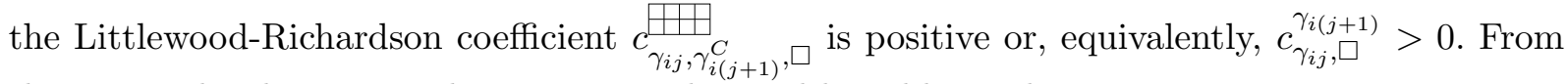
the Pieri rule, this means that $\gamma_{i(j+1)}$ is obtained by adding a box to $\gamma_{i j}$.

The same argument shows that $\gamma_{(i-1) j}$ is obtained by adding a box to $\gamma_{i j}$.

Finally, we check condition (2). Pick a curve $X$ with real points marked by $[i, j)$ in circular order, plus one more point between $j-1$ and $i$. Pick another curve $Z$ with real points marked by $[j+1, i-1)$ in circular order, plus one more point between $i-2$ and $j+1$. Glue $X$ and $Z$ to $\mathbb{R P}^{1}$ at 0 and $\infty$. Also mark two more points of this central $\mathbb{R P}^{1}$, at 1 and at $\tau \in(-\infty, 0)$, and label these points $j$ and $i-1$. As $\tau$ varies through $[\infty, 0]$, this sweeps out a path through $\sigma$.

On the central component, 0 and $\infty$ are labeled by the partitions $\gamma_{i j}$ and $\gamma_{(i-1)(j+1)}^{C}$. The points 1 and $\tau$ are labeled by $\square$. We want to compute the labels that wind up on the additional node formed when $\tau$ collides with 0 , and when $\tau$ collides with $\infty$. According to Theorem 4.4, they are unequal, as desired.

The main result of this section is the following:

THEOREM 7.3. Over any maximal face $\sigma$ of $\bar{M}_{0, r}(\mathbb{R})$, the above construction gives a bijection between the preimage faces of $\mathcal{S}(\mathbb{R})$ and cylindrical growth diagrams.

Proof of Theorem 7.3. We recall the notation $(s(1), s(2), \ldots, s(r))$ for the circular ordering of $[r]$ corresponding to $\sigma$. We will use the notation $s(i)$ for $i$ an arbitrary integer, meaning to reduce $i$ modulo $r$.

Fix a path $\delta=\left(\left(i_{0}, j_{0}\right),\left(i_{1}, j_{1}\right), \ldots,\left(i_{r}, j_{r}\right)\right)$ through $\mathbb{I}$. As shown in Lemma 6.1 , there is a bijection between cylindrical growth diagrams and maps $\delta \rightarrow \Lambda$ which start at $\emptyset$ and grow to $\boxplus$, adding one box at a time. Such a map $\delta \rightarrow \Lambda$ can, clearly, be thought of as a standard Young tableau of shape $\boxplus$; we recall the notation $\operatorname{SYT}(d, n)$ for the set of such standard tableaux. So we must show that each standard Young tableau occurs for exactly one face of $\mathcal{S}(\mathbb{R})$, over the fixed face of $\bar{M}_{0, r}(\mathbb{R})$.

Define a permutation $\pi$ of $[r]$ as follows: $\pi(k)$ is $s\left(i_{k}\right)$ if $i_{k}=i_{k-1}-1$ and $\pi(k)=s\left(j_{k-1}\right)$ if $j_{k}=j_{k-1}+1$. To see that this is a permutation, note that the quantity $j_{k}-i_{k}$ increases by 1 every time $k$ increases. So, for each $k$, we add one more element to the interval $\left[i_{k}, j_{k}\right)$. When we reach $r$, we have $j_{r}-i_{r}=r$, so the interval $\left[i_{r}, j_{r}\right)$ will contain every equivalence class modulo $r$ exactly once. The permutation $\pi$ lists the elements of $\left[i_{r}, j_{r}\right)$ in the order they were added. In other words, $\pi$ is constructed so that $\pi([k])=\left[i_{k}, j_{k}\right)$. 


\section{DAVID E SPEYER}

We will now describe a particular stable curve $X$, and will write $x$ for the corresponding point in $\bar{M}_{0, r}(\mathbb{R})$. The reader may want to look at Figure 6 , where we show $X$ for the path in Figure 4, and at the discussion after this proof.

The curve $X$ has $r-2$ components, $X_{1}, X_{2}, \ldots, X_{r-2}$, with nodes connecting $X_{i}$ and $X_{i+1}$. The component $X_{1}$ has marked points $z(\pi(1))$ and $z(\pi(2))$; the component $X_{r-2}$ has marked points $z(\pi(r-1))$ and $z(\pi(r))$; for $2 \leqslant i \leqslant r-3$, the component $X_{i}$ contains the marked point $z(\pi(i+1))$. So the node between $X_{k-1}$ and $X_{k}$ separates the marked points labeled by $\left[i_{k}, j_{k}\right)$ and those labeled by $\left[j_{k}, i_{k}\right)$. Thus, knowing the values of $\gamma_{i_{k} j_{k}}$ is equivalent to knowing the partitions labeling the nodes in the fiber of $\mathcal{S}$ above $x$.

By Theorem 1.2, the fiber of $\mathcal{S}$ over $x$ corresponds to all ways of labeling the nodes of $X$ by partitions, and of choosing a solution to the corresponding Schubert problem for each component of $X$. In this case, each of these Schubert problems is of the form $\lambda \cdot \mu \cdot \square$. By the Pieri rule, $c_{\lambda \mu \square}$ is 1 if $\mu^{C}$ is $\lambda$ with a box added on, and 0 otherwise. So we only get a point of $\mathcal{S}$ over $x$ if the labels of the nodes form a chain in $\operatorname{SYT}(d, n)$, and in this case we get one point. So we have a bijection from $\operatorname{SYT}(d, n)$ to the fiber of $\mathcal{S}$ over $x$.

We now note that $x$ is a vertex of $\sigma$. For every node of $X$, the marked points lying to one side of this node form a cyclic interval in the circular ordering $s$, so the topology of $X$ occurs as a limit of the circular ordering topology given by $s$.

Fix a particular growth diagram $\gamma$ for which we want to show that there is a unique face labeled $\gamma$. Let $T$ be the standard Young tableau found by restricting $\gamma$ to the path $\delta$.

If $\tau$ is any maximal face of $\mathcal{S}(\mathbb{R})$, lying above $\sigma$ and labeled by $\gamma$, then let $y$ be the point of $\tau$ above $x$. Then $y$ is labeled by $T$. Conversely, if there is some $y$ above $x$ labeled by $T$, then, since $\mathcal{S}(\mathbb{R}) \rightarrow \bar{M}_{0, r}$ is a covering map, there is a unique maximal face $\tau$ of $\mathcal{S}(\mathbb{R})$ lying above $\sigma$ and containing $y$. Letting $\gamma^{\prime}$ be the label of $\tau$, we see that $\gamma$ and $\gamma^{\prime}$ coincide along the path $\delta$ and are hence equal, by Lemma 6.1.

So maximal faces of $\mathcal{S}(\mathbb{R})$ labeled by $\gamma$ are in bijection with points lying above $x$ labeled by $T$, and there is one of those.

Having proved our main theorem for the section, we discuss consequences.

\subsection{Periodicity of cylindrical growth diagrams}

The following purely combinatorial result is [Hai92, Theorem 4.4]. We provide a geometric proof.

Proposition 7.4. In any cylindrical growth diagram, we have $\gamma_{i j}=\gamma_{(r+j) i}^{C}=\gamma_{(r+j)(r+j)}$.

Proof. We now know that every cylindrical growth diagram comes from an actual geometric face of $\mathcal{S}$ lying over $\sigma$. Let $X$ be a curve in that face where one component contains the marked points $[i, j)$ and the other contains the marked points $[j, i)=[j, i+r)$. Then $\gamma_{i j}$ and $\gamma_{(r+j) i}$ are the two partitions labeling that node from opposite sides, so they are complementary, and $\gamma_{i j}$ and $\gamma_{(i+r)(j+r)}$ are two names for the partition labeling the same node.

This now explains the term cylindrical: If we quotient $\mathbb{I}$ by $(i, j) \equiv(i+r, j+r)$, it is natural to draw the corresponding diagrams on a cylinder.

Figure 6 shows the caterpillar curve corresponding to the path in Figure 4. The rows of Figure 4 are numbered 1 through 7 , so that the path travels through positions $(4,4),(3,4)$, $(3,5),(3,6),(2,6),(2,7),(2,8)$. The marked points are shown as solid dots, and one side of each 


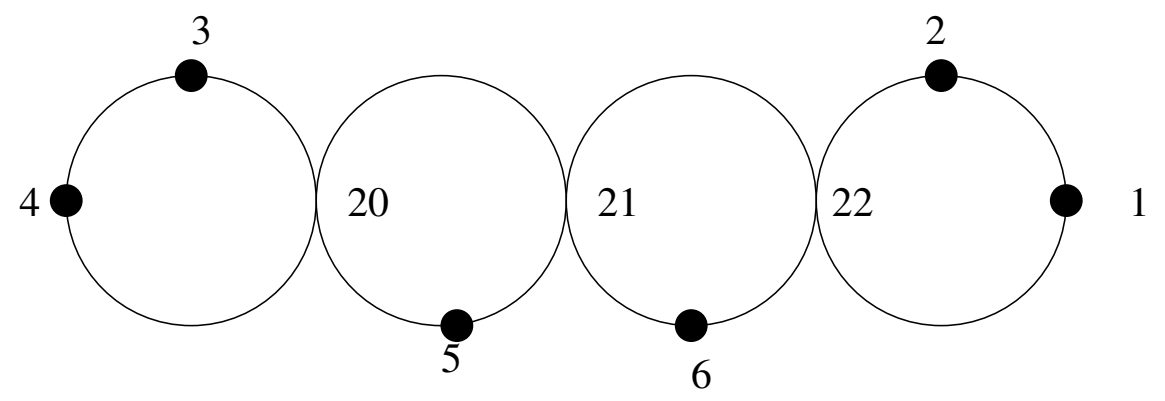

FiguRE 6. A caterpillar curve, corresponding to the path in Figure 4

node is labeled with the partition coming from Figure 4. (The complementary partition, labeling the opposite side of the node, has been omitted to reduce clutter.)

\subsection{The missing computation from Theorem 4.4}

We now verify that the points (3) and (6) in Theorem 4.4 are ordered as claimed.

Lemma 7.5. Let $\gamma_{\bullet}$ be a cylindrical growth diagram. As we go from $\gamma_{i j}$ to $\gamma_{i(j+1)}$ to $\gamma_{i(j+2)}$, we add two boxes. If the second box added is northeast of the first, then $\gamma_{j(j+2)}=(2)$. If the second box is added southwest of the first, then $\gamma_{j(j+2)}=(1,1)$.

Proof. Suppose that the second box is added northeast of the first. Then I claim that, for every $k$ between $j-r+2$ and $j$, when we go from $\gamma_{k j}$ to $\gamma_{k(j+1)}$ to $\gamma_{k(j+2)}$, the second box is added northeast of the first. This is just checked by looking what the recursion does when we change from the path $\left(\gamma_{(k+1) j}, \gamma_{k j}, \gamma_{k(j+1)}, \gamma_{k(j+2)}\right)$ to $\left(\gamma_{(k+1) j}, \gamma_{(k+1)(j+1)}, \gamma_{(k+1)(j+2)}, \gamma_{k(j+2)}\right)$.

In particular, along the path $\left(\gamma_{j j}, \gamma_{j(j+1)}, \gamma_{j(j+2)}\right)$, we add the second box northeast of the first. But $\gamma_{j j}=\emptyset$ and $\gamma_{j(j+1)}=\square$, so the only way to add a square to the northeast is for $\gamma_{j(j+2)}$ to be (2), a horizontal domino. Similar arguments do the other case.

Conceptual verification of the missing point from Theorem 4.4. We work over the face of $\bar{M}_{0, r}$ corresponding to the circular ordering $1,2, \ldots, r$.

Take any cylindrical growth diagram with $\left(\gamma_{i j}, \gamma_{i(j+1)}, \gamma_{i(j+2)}\right)=\left(\lambda, \kappa_{1}, \mu^{C}\right)$. Consider a stable curve with three components: a central component with markings $j$ and $j+1$, and two other components joined to this one, one with marked points indexed by $[i, j)$ and the other with marked points labeled by $[j+2, i)$. Let the component marked by $[i, j)$ be attached to the central component at 0 ; let the marked point $j$ be $\tau$; let the marked point $j+1$ be 1 ; and let the component marked by $[j+2, i)$ be attached at $\infty$, with $0<\tau<1<\infty$. When $\tau$ collides with 0 , a new component bubbles off and where that new component is attached to the central component, we see the marking $\kappa_{1}$. When $\tau$ collides with 1 , a new component bubbles off and where that new component is attached to the central component, we see $\gamma_{j(j+2)}$, which, by Lemma 7.5 , is (2).

If we let $\tau$ go all the way around the central component, we recover the monodromy from Theorem 4.4. In particular, we have just seen that, as $\tau$ goes from 0 to 1 , the point above 0 where the node is labeled by $\kappa_{1}$ goes to $(2)$. Similarly, the point above 0 where the node is labeled by $\kappa_{2}$ goes to $(1,1)$. 


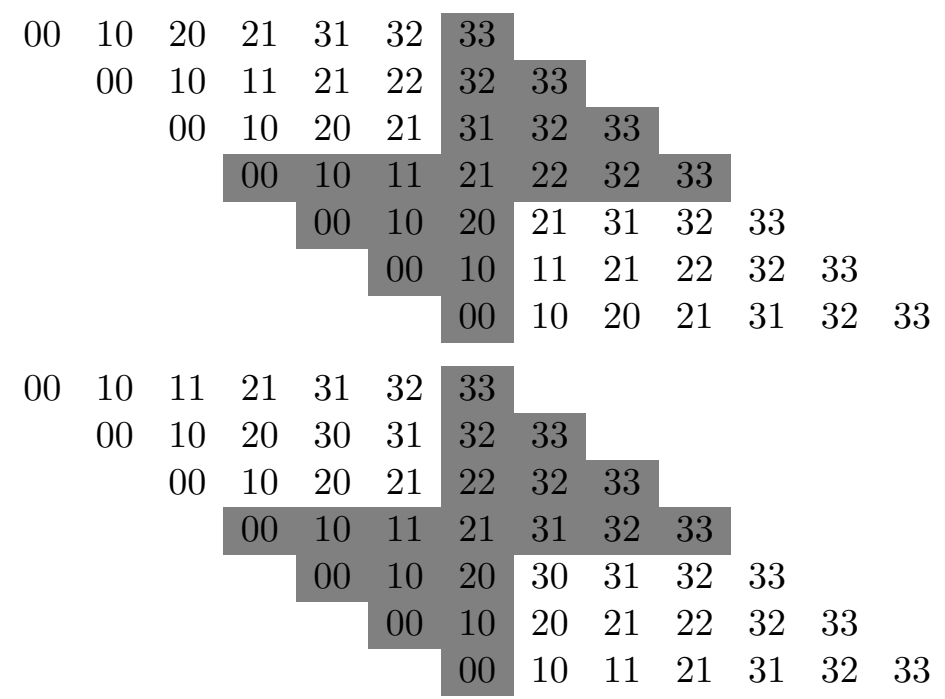

Figure 7. Crossing a wall

\subsection{Effect of wall crossing}

We now understand how to index the maximal faces of $\mathcal{S}$ : They correspond to pairs of (1) a circular ordering of $[r]$, up to reflection and (2) a cylindrical growth diagram. Note that, if we shift the circular ordering, we translate the cylindrical growth diagram, changing $(i, j)$ to $(i+k, j+k)$. If we reflect the circular order, we reflect the cylindrical growth diagram, changing $(i, j)$ to $(k-j, k-i))$.

We now discuss how these maximal faces are glued together. Let $p<q$ be distinct elements of $[r]$. Let $\hat{\sigma}$ be the adjacent face of $\bar{M}_{0, r}(\mathbb{R})$ where we reverse the order of $s(q), s(q+1), \ldots$, $s(p-1)$ (indices are cyclic modulo $r$ ). Let $\gamma_{\bullet \bullet}$ be a cylindrical growth pattern indexing a face over $\sigma$, and let $\hat{\gamma}_{\bullet \bullet}$ index the face over $\hat{\sigma}$.

Proposition 7.6. For $p \leqslant i \leqslant j \leqslant q$, we have $\gamma_{i j}=\hat{\gamma}_{i j}$. For $q \leqslant i \leqslant j \leqslant p+r$, we have $\gamma_{i j}=\hat{\gamma}_{(p+q-j-1)(p+q-i-1)}$.

Proof. The nodes in question can occur on curves which live on the wall between $\sigma$ and $\hat{\sigma}$. The partition labeling the node is the same in both cases, but which element of $\mathbb{I}$ indexes that node will differ if $i$ and $j$ are in $[q, p)$, because the elements in $[q, p)$ are reversed when we cross the wall.

The entries $\hat{\gamma}_{i j}$ for other $(i, j)$ are uniquely determined by the values we have described, using Lemma 6.1. I do not have a simple description of the other entries.

We could have equally well described crossing the wall by reversing $[p, q)$, in which case the circular growth diagram $\hat{\gamma}$ would differ by the appropriate reflection.

The top half of Figure 7 is the cylindrical growth diagram of Figure 4 . The bottom half shows the new growth diagram we obtain when we cross a wall reversing the order of $(s(4), s(5), s(6))$. Proposition 7.6 tells us that the southwest shaded triangle in the bottom diagram is identical to that in the top diagram, and that the northeast shaded triangles are reflections of each other over a line of slope 1. (This reflection interchanges the entries 22 and 31.) The unshaded entries must then be computed recursively. 


\section{SCHUBERT PROBlEMS FROM STABLE CURVES}

\subsection{The case $d=2$ and the work of Eremenko and Gabrielov}

In this section, we consider the special case $d=2$. The number of standard Young tableaux of shape $2 \times(n-2)$ is the Catalan number $C_{n}=(2 n-4) ! /(n-2) !(n-1)$ !, and so this is also the number of cylindrical growth diagrams. The bijection between cylindrical growth diagrams and standard Young tableaux requires choosing a path through $\mathbb{I}$.

Consider a sequence of points $p_{1}, p_{2}, \ldots, p_{2 n-4}$ arranged around a circle; note that $2 n-4=r$. A noncrossing matching is a partition of $[r]$ into $n-2$ sets of size 2 such that, if $\{i, j\}$ and $\{k, \ell\}$ are elements of the matching, then the chords $\overline{p_{i} p_{j}}$ and $\overline{p_{k} p_{\ell}}$ don't cross. $C_{n}$ also counts the number of noncrossing matchings.

The following is a bijection from noncrossing matchings to cylindrical growth diagrams: Among $\left\{p_{i}, p_{i+1}, \ldots, p_{j-1}\right\}$, let there be $s$ arcs connecting one of these points to another, and let there be $t$ arcs connecting one of the points to a point in $\left\{p_{j}, p_{j+1}, \ldots, p_{i-1}\right\}$, so $j-i=2 s+t$. Take $\gamma_{i j}=(s+t, s)$.

This bijection was published in [PPR09], where it is credited to White. More precisely, in [PPR09], this is described as a bijection between noncrossing matchings and standard Young tableaux which carries the rotational symmetry of matchings to the action of promotion on tableaux. A cylindrical growth diagram records all of the promotions of a given tableaux.

Observe that there is an arc of the matching from $p_{i}$ to $p_{j}$ if and only if, for some $s$, we have $\gamma_{(i+1) j}=(s, s)$ and $\gamma_{i(j+1)}=(s+1, s+1)$. So $\gamma$ is determined by knowing the set of $(i, j)$ for which $\left(\gamma_{(i+1) j}, \gamma_{i(j+1)}\right)$ is of the form $((s, s),(s+1, s+1))$. The reader may like to verify that the growth diagram of Figure 4 corresponds to the matching $\{1,2\},\{3,4\},\{5,6\}$.

We introduce the abbreviation $\mathcal{S}_{0}$ for the part of $\mathcal{S}(\mathbb{R})$ lying above $M_{0, r}(\mathbb{R})$. Eremenko and Gabrielov [EG02] describe a beautiful way to decompose $\mathcal{S}_{0}$ into cells labeled by noncrossing matchings. We will show that our decomposition corresponds to theirs under the above combinatorial recipe.

Eremenko and Gabrielov's construction uses the connection to the Wronski problem discussed in the introduction. Take a point $w$ in $\mathcal{S}_{0}$. Let $z_{1}, z_{2}, \ldots, z_{r} \in \mathbb{R} \mathbb{P}^{1}$ be the marked points of the point of $M_{0, r}(\mathbb{R})$ below $w$. Identify $\mathbb{R}^{n}$ with $\mathbb{R}[x]_{\leqslant n-1}$, the space of polynomial of degree $\leqslant n-1$; let $w$ be spanned by $p(x)$ and $q(x) \in \mathbb{R}[x]_{\leqslant n-1}$. Let $\phi: \mathbb{P}^{1} \rightarrow \mathbb{P}^{1}$ be the rational map $z \mapsto p(z) / q(z)$. The Schubert condition $\Omega\left(\square, z_{i}\right)$ means that $\phi$ has a critical point at $z_{i}$.

Let $W:=\phi^{-1}\left(\mathbb{R P}^{1}\right)$, a one dimensional CW complex embedded in $\mathbb{C P}^{1}$. Let $N$ and $S$ be the two hemispheres into which $\mathbb{C P}^{1}$ is divided by $\mathbb{R P}^{1}$.

By the MTV theorem (which was proved in [EG02] in this case), $\phi$ is defined over $\mathbb{R}$. This implies that $\mathbb{R P}^{1} \subset W$ and that $W \cap N$ and $W \cap S$ are complex conjugates of each other. $W \cap N$ is a collection of noncrossing arcs, terminating at the $z_{i}$. The main result of [EG02] is that, for any $\left(z_{1}, z_{2}, \ldots, z_{r}\right)$ in $M_{0, r}(\mathbb{R})$, and any noncrossing matching, there is precisely one element of $\mathcal{S}_{0}$ where $W$ has the topology given by that matching.

THEOREM 7.7. Let $\tau$ be the maximal face of $\mathcal{S}$ containing $w$; let $\gamma$ be the cylindrical growth diagram indexing $\tau$; let $M$ be the corresponding noncrossing matching. Then $N \cap W$ has the same topology as $M$.

Proof. We will show that, if $\left(\left(\gamma_{(i+1) j}, \gamma_{i(j+1)}\right)\right.$ is of the form $((s, s),(s+1, s+1))$, then $W$ contains an arc joining $z_{i}$ and $z_{j}$. By the previous observations, there is a $\gamma_{i j}$ of the form $(s, s)$ if and only if there is an arc of $M$ from $i$ to $j$, and we know that $M$ and $N \cap W$ both have $r / 2=n-2 \operatorname{arcs}$, so this will show that $M$ and $N \cap W$ have the same connectivity. 


\section{DAVID E SPEYER}

Let $\left(\left(\gamma_{(i+1) j}, \gamma_{i(j+1)}\right)\right.$ be of the form $((s, s),(s+1, s+1))$. Note that the topology of $W$ is constant on connected components of $\mathcal{S}_{0}$. So we can let $w$ approach the codimension 2 face of $\tau$ corresponding to stable curves with three components: one which contains $\left\{z_{k}: k \in(i, j)\right\}$, one which contains $\left\{z_{\ell}: \ell \in(j, i)\right\}$ and one, the middle component, which contains $z_{i}$ and $z_{j}$. Call these components $C_{1}, C_{2}, C_{3}$. In the limit where $w$ lies in this codimension 2 face, we can consider $w$ as a point in $G(2, n)_{C_{1}} \times G(2, n)_{C_{2}} \times G(d, n)_{C_{3}}$, as in Section 3 .

Let $w_{2}$ be the $C_{2}$-component of $w$. As above, let $w_{2}$ be spanned by $p_{2}$ and $q_{2} \in \mathbb{R}[x]_{\leqslant n-1}$. Choose coordinates on $C_{2}$ such that the node joining $C_{1}$ and $C_{2}$ is at 0 , and the node joining $C_{2}$ and $C_{3}$ at $\infty$. The condition $\gamma_{(i+1) j}=(s, s)$ means that $p_{2}$ and $q_{2}$ vanish to order $s$ at 0 ; the condition $\gamma_{i(j+1)}=(s+1, s+1)$ means that $\operatorname{deg} p_{2}=\operatorname{deg} q_{2}=s+2$. So $\phi_{2}=p_{2} / q_{2}$ is the unique degree 2 rational map with critical points $z_{i}$ and $z_{j}$. For this rational function, $\phi_{2}^{-1}\left(\mathbb{R P}^{1}\right)$ is four $\operatorname{arcs}$ joining $z_{i}$ and $z_{j}$, two of them passing through 0 and $\infty$.

When we move $w$ off of the codimension 2 face, so that the node smooths, the two arcs from $z_{i}$ to $z_{j}$ which avoid the nodes remain. So $W$ has an arc from $z_{i}$ to $z_{j}$, as desired.

REMARK 7.8. One might hope for a similar construction in the case $d=3$. Kuperberg [Kup96] defines $A_{2}$-webs, the number of which is the same as the number of standard Young tableaux of shape $3 \times(n-3)$. These $A_{2}$-webs have rotational symmetry, just as noncrossing matchings do. Khovanov-Kuperberg [KK99] present a bijection from webs to Young tableaux. Translated into a bijection from webs to cylindrical growth diagrams, the map of [KK99] is the following: Take a minimal cut path $\delta$ from $i$ to $j$, as defined in [KK99, Section 5]. Then $\gamma_{i j}=(s+t+u, s+t, s)$ where $s$ is the number of arcs crossing $\delta$ from left to right, $u$ is the number of arcs crossing $\delta$ from right to left, and $t$ is determined by the condition $j-i=3 s+2 t+u$. It would be fascinating to find a geometric interpretation of webs similar to the interpretation Eremenko and Gabrielov find for noncrossing matchings.

\section{Dual equivalence}

In the last section, we gave an explicit indexing set for the facets of $\mathcal{S}(\square, \square, \cdots, \square)$ lying over a fixed facet of $\bar{M}_{0, r}$. In the next section, we will generalize this to an arbitrary Schubert problem $\mathcal{S}\left(\lambda_{1}, \lambda_{2}, \ldots, \lambda_{r}\right)$ with $\sum\left|\lambda_{i}\right|=d(n-d)$. Our generalization uses the language of dual equivalence, so we rapidly review this topic. Our reference is [Hai92]

Let $\mathbb{J}$ be a a subset of $\mathbb{Z}^{2}$ of the form $[a, b] \times[c, d]$. A growth diagram is a map $\gamma: \mathbb{J} \rightarrow \Lambda$ which satisfies conditions (1) and (2) in the definition of a cylindrical growth diagram. In other words, a growth diagram is a possible rectangular region within a cylindrical growth diagram. (Historically, growth diagrams are the standard notion; the term "cylindrical growth diagram" is my own.)

Note that, if we know the partitions $\gamma_{i j}$ for $(i, j) \in\{b\} \times[c, d] \cup[a, b] \times\{d\}$, then all of the others are recursively determined. We introduce terminology for this idea: Let $\mu \subset \nu \subset \pi$ be a chain of partitions, let $\delta_{\bullet} \in \mathrm{SYT}(\nu / \mu)$ and $\epsilon_{\bullet} \in \mathrm{SYT}(\pi / \nu)$. Set $m=|\mu|, n=|\nu|$ and $p=|\pi|$, so $\delta$ and $\epsilon$ are chains of length $n-m$ and $p-n$. Let $\gamma:[-(p-n), 0] \times[m, n] \rightarrow \Lambda$ be the unique growth diagram with $\gamma_{0(m+j)}=\delta_{j}$ and $\gamma_{(-i) n}=\epsilon_{i}$. Let $\delta^{\prime}$ be the tableau $\delta_{j}^{\prime}=\gamma_{(n-p)(m+j)}$ and let $\epsilon^{\prime}$ be the tableau $\epsilon_{i}^{\prime}=\gamma_{(-i) 0}$. So $\delta^{\prime} \in \operatorname{SYT}\left(\pi / \nu^{\prime}\right)$ and $\epsilon^{\prime} \in \operatorname{SYT}\left(\nu^{\prime} / \mu\right)$ for some $\nu^{\prime}$. We say that $\left(\epsilon^{\prime}, \delta^{\prime}\right)$ is the result of shuffling $(\delta, \epsilon)$. The growth diagram recursion is symmetric in switching $-i$ and $j$, so shuffling $\left(\epsilon^{\prime}, \delta^{\prime}\right)$ restores the original $(\delta, \epsilon)$.

We define two equivalence relations: slide equivalence and dual equivalence. 


\section{SCHUBERT PROBlEMS FROM STABLE CURVES}

Slide equivalence is the finest equivalence relation such that if $(\delta, \epsilon)$ shuffles to $\left(\epsilon^{\prime}, \delta^{\prime}\right)$, then $\delta$ is slide equivalent to $\delta^{\prime}$ and $\epsilon$ is slide equivalent to $\epsilon^{\prime}$.

Two dual equivalent tableaux will always have the same shape. Dual equivalence is the coarsest equivalence relation on $\operatorname{SYT}(\nu / \mu)$ satisfying the following: Let $\delta_{1}$ and $\delta_{2} \in \operatorname{SYT}(\nu / \mu)$ be dual equivalent, and let $\epsilon$ be any element of $\operatorname{SYT}(\pi / \nu)$, for any $\pi \supseteq \nu$. Write $\left(\epsilon_{1}^{\prime}, \delta_{1}^{\prime}\right)$ and $\left(\epsilon_{2}^{\prime}, \delta_{2}^{\prime}\right)$ for the results of shuffling $\epsilon$ past $\delta_{1}$ and $\delta_{2}$. Then $\epsilon_{1}^{\prime}=\epsilon_{2}^{\prime}$.

The following are the key results about dual and slide equivalence:

Proposition 8.1 ([Hai92, Theorem 2.13]). If two tableaux are both dual and slide equivalent, then they are equal.

Proposition 8.2 ([Hai92, Corollary 2.5]). Any two tableaux in $\operatorname{SYT}(\mu / \emptyset)$ are dual equivalent to each other.

Proposition 8.3. If $\delta_{1}$ and $\delta_{2}$ are dual equivalent, and $\left(\epsilon^{\prime}, \delta_{1}^{\prime}\right),\left(\epsilon^{\prime}, \delta_{2}^{\prime}\right)$ are the results of shuffling the $\delta_{i}$ with some $\epsilon$, then $\delta_{1}^{\prime}$ and $\delta_{2}^{\prime}$ are dual equivalent.

Proof. Immediate corollary of [Hai92, Lemma 2.3 and Theorem 2.6].

Proposition 8.4. Any slide equivalence class contains a unique tableaux $\delta$ of straight shape.

Proof. This is the "first fundamental theorem of jeu de taquin". As Haiman sketches in the final paragraphs of [Hai92, Section 2], it follows from Proposition 8.2.

Given an arbitrary tableau $\epsilon$, we will say that $\epsilon$ rectifies to $\delta$ if $\delta$ is the unique tableau of straight shape in its slide equivalence class. In this context, we will write $\operatorname{rshape}(\epsilon)$ for shape $(\delta)$. If $\epsilon_{1}$ and $\epsilon_{2}$ are dual equivalent, then $\operatorname{rshape}\left(\epsilon_{1}\right)=\operatorname{rshape}\left(\epsilon_{2}\right)$.

Proposition 8.5. Given $\epsilon \in \operatorname{SYT}(\mu / \emptyset)$, and $\delta$ a tableau with $\operatorname{rshape}(\delta)=\mu$, there is a unique tableau $\phi$ which is slide equivalent to $\epsilon$ and dual equivalent to $\delta$.

Proof. Uniqueness is an immediate consequence of Proposition 8.1; we must show existence.

Let shape $(\delta)=\nu / \lambda$. Choose $\alpha \in \operatorname{SYT}(\lambda)$. Let $\left(\epsilon_{2}, \beta\right)$ be the result of shuffling $(\alpha, \delta)$. So shape $\left(\epsilon_{2}\right)=\operatorname{rshape}(\delta)=\mu$ and thus shape $(\beta)=\nu / \mu$; we see that it makes sense to shuffle $(\epsilon, \beta)$. Let the result of shuffling $(\epsilon, \beta)$ be $(\psi, \phi)$. By definition, $\epsilon$ is slide equivalent to $\phi$. By Proposition 8.2, $\epsilon$ and $\epsilon_{2}$ are dual equivalent so, by Proposition 8.3, $\delta$ and $\phi$ are dual equivalent.

We now introduce shuffling of dual equivalence classes.

Proposition 8.6. Let $\delta \in \operatorname{SYT}(\nu / \mu)$ and let $\epsilon \in \operatorname{SYT}(\pi / \nu)$, for some $\mu \subset \nu \subset \pi$. Let $(\delta, \epsilon)$ shuffle to $\left(\epsilon^{\prime}, \delta^{\prime}\right)$. The dual equivalence classes of $\delta^{\prime}$ and $\epsilon^{\prime}$ are determined by the dual equivalence classes of $\delta$ and $\epsilon$.

Proof. Let $\epsilon_{1}$ and $\epsilon_{2}$ be dual equivalent. Let $\left(\epsilon_{1}^{\prime}, \delta^{\prime}\right)$ and $\left(\epsilon_{2}^{\prime}, \delta^{\prime}\right)$ be the results of shuffling $\left(\delta, \epsilon_{1}\right)$ and $\left(\delta, \epsilon_{2}\right)$. (The second entry is the same in both cases by the definition of dual equivalence.) Then $\delta^{\prime}$ is tautologically dual equivalent to itself, and $\epsilon_{1}^{\prime}$ and $\epsilon_{2}^{\prime}$ are dual equivalent by Proposition 8.3.

So replacing $\epsilon$ by a dually equivalent tableau does not change the dual equivalence classes of $\delta^{\prime}$ and $\epsilon^{\prime}$. Similarly, replacing $\delta$ by a dually equivalent tableau does not change the dual equivalence classes of $\delta^{\prime}$ and $\epsilon^{\prime}$. Combining these two facts, we have the claim.

Thus, we may speak of shuffling two dual equivalence classes, to obtain another pair of dual equivalence classes. 


\section{DAVID E SPEYER}

\section{Dual equivalence classes of cylindrical growth diagrams}

Let $0<d<n$ and take $r \geqslant 3$; we are no longer setting $r=d(n-d)$. Fix partitions $\lambda_{1}, \lambda_{2}, \ldots$, $\lambda_{r}$ obeying $\sum_{k=1}^{r}\left|\lambda_{k}\right|=d(n-d)$. Let $\mathbb{I}$, as in Section 6 , be $\left\{(k, \ell) \in \mathbb{Z}^{2}: k \leqslant \ell \leqslant k+r\right\}$.

A dual equivalence cylindrical growth diagram, which we will abbreviate to decgd, consists of two pieces of data:

(1) a map $\gamma: \mathbb{I} \rightarrow \Lambda$ such that $\gamma_{k \ell}$ is contained in $\gamma_{k(\ell+1)}$ and $\gamma_{(k-1) \ell}$, and

(2) for every $(k, \ell) \in \mathbb{I}$ with $\ell-k<r$, a choice $a(k, \ell)$ and $b(k, \ell)$ of dual equivalence classes in $\operatorname{SYT}\left(\gamma_{k(\ell+1)} / \gamma_{k \ell}\right)$ and $\operatorname{SYT}\left(\gamma_{(k-1) \ell} / \gamma_{k \ell}\right)$, respectively,

such that

(1) $\gamma_{k k}=\emptyset$ and $\gamma_{k(k+r)}=\boxplus$.

(2) If we shuffle the dual equivalence classes $a(k, \ell)$ and $b(k, \ell+1)$, we obtain the dual equivalence classes $b(k, \ell)$ and $a(k-1, \ell)$.

We will use indices $(k, \ell)$ for dual equivalence cylindrical growth diagrams, and reserve $(i, j)$ for ordinary cylindrical growth diagrams. Note that, since the shape of $a(k, \ell)$ is $\gamma_{k(\ell+1)} / \gamma_{k \ell}$, the dual equivalence classes $a(k, \ell)$ and $b(k, \ell)$ determine the map $\gamma$.

The shape of the decgd $\gamma$ is the sequence of partitions $\left(\gamma_{01}, \gamma_{12}, \ldots, \gamma_{(r-1) r}\right)$.

The main result of this section is that, over the face of $\bar{M}_{0, r}(\mathbb{R})$ associated to the circular ordering $(s(1), \ldots, s(r))$, the maximal faces of $\mathcal{S}\left(\lambda_{1}, \ldots, \lambda_{r}\right)(\mathbb{R})$ are indexed by decgds of shape $\left(\lambda_{s(1)}, \ldots, \lambda_{s(r)}\right)$.

Intuitively, we want decgds to be equivalence classes of cylindrical growth diagrams. We now explain how to map a cylindrical growth diagram $\widetilde{\gamma}$ to a decgd $\gamma$; we call $\gamma$ the restriction of $\widetilde{\gamma}$.

Set $\widetilde{r}=d(n-d)$ and $\widetilde{\mathbb{I}}=\left\{(i, j) \in \mathbb{Z}^{2}: i \leqslant j \leqslant i+\widetilde{r}\right\}$. Let $\widetilde{\gamma}: \widetilde{\mathbb{I}} \rightarrow \Lambda$ be a cylindrical growth diagram. Let $\iota: \mathbb{Z} \rightarrow \mathbb{Z}$ be such that $\iota(\ell)-\iota(\ell-1)=\left|\lambda_{\ell}\right|$, with the index $\ell$ periodic modulo $r$ on the right-hand side. So $\iota(i+r)-\iota(i)=\sum_{\ell=1}^{r}\left|\lambda_{\ell}\right|=\widetilde{r}$. Let $\gamma_{k \ell}=\widetilde{\gamma}_{\iota(k) \iota(\ell)}$; let $a(k, \ell)$ be the dual equivalence class of $\widetilde{\gamma}_{\iota(k) \iota(\ell)}, \widetilde{\gamma}_{\iota(k)(\iota(\ell)+1)}, \widetilde{\gamma}_{\iota(k)(\iota(\ell)+2)}, \ldots, \widetilde{\gamma}_{\iota(k) \iota(\ell+1)}$; and let $b(k, \ell)$ be the dual equivalence class of $\widetilde{\gamma}_{\iota(k) \iota(\ell)}, \widetilde{\gamma}_{(\iota(k)-1) \ell}, \ldots, \widetilde{\gamma}_{\iota(k-1) \iota(\ell)}$.

Proposition 9.1. Every decgd is the restriction of a cylindrical growth diagram. More specifically, choose a path $\delta$ through $\mathbb{I}$ and choose lifts $\tilde{a}(k, \ell)$ and $\tilde{b}(k, \ell)$ of $a(k, \ell)$ and $b(k, \ell)$, respectively, along $\gamma$; then there is a unique way to extend this lifting to cylindrical growth diagram. The number of decgd's of shape $\left(\lambda_{1}, \lambda_{2}, \ldots, \lambda_{r}\right)$ is the Littlewood-Richardson coefficient $c_{\lambda_{1} \lambda_{2} \cdots \lambda_{r}}$.

Proof. Choose a path $\delta$ through $\mathbb{I}$ and choose lifts $\tilde{a}(k, \ell), \tilde{b}(k, \ell)$ of $a(k, \ell)$ and $b(k, \ell)$, respectively, along this path. By Lemma 6.1, these lifts extend uniquely to a cylindrical growth diagram $\tilde{\gamma}$; what we need to know is that $\tilde{\gamma}$ restricts to the original $\gamma$. But this is a simple inductive statement from the definition of shuffling dual equivalence classes.

We now establish the claim about the Littlewood-Richardson coefficient. For notational convenience, we choose our starting path $\delta$ to be $(0,0) \rightarrow(0,1) \rightarrow(0,2) \rightarrow \cdots \rightarrow(0, r)$. We abbreviate $\gamma_{0 j}$ to $\mu_{j}$ and $a(0, j)$ to $a(j)$. As in Proposition 6.1, there is a unique $\gamma$ extending any choice of partitions $\mu_{j}$ and dual equivalence classes $a(j) \in \operatorname{SYT}\left(\mu_{j+1} / \mu_{j}\right)$. This unique $\gamma$ will have shape $\left(\lambda_{1}, \lambda_{2}, \ldots, \lambda_{r}\right)$ if and only if $\operatorname{rshape}(a(j))=\lambda_{j}$. The number of dual equivalence classes in $\operatorname{SYT}\left(\mu_{j+1} / \mu_{j}\right)$ with rectification shape $\lambda_{j+1}$ is $c_{\lambda_{j+1} \mu_{j}}^{\mu_{j+1}}$ (see [Sta99, Theorem A.1.3.1] 


\section{SCHUBERT PROBlems FROM STABLE CURVES}

and Proposition 8.5.) So the number of decgd's of shape $\left(\lambda_{1}, \lambda_{2}, \ldots, \lambda_{r}\right)$ is

$$
\sum_{\emptyset=\mu_{0} \subset \mu_{1} \subset \mu_{2} \subset \cdots \subset \mu_{r}=\boxplus} c_{\mu_{0}, \lambda_{1}}^{\mu_{1}} c_{\mu_{1}, \lambda_{2}}^{\mu_{2}} \cdots c_{\mu_{r-1}, \lambda_{r}}^{\mu_{r}}=c_{\lambda_{1} \lambda_{2} \cdots \lambda_{r}}^{\boxplus}
$$

by the associativity of the Littlewood-Richardson coefficients.

We now describe the indexing of maximal faces of $\mathcal{S}\left(\lambda_{1}, \ldots, \lambda_{r}\right)(\mathbb{R})$ by decgds. We embed $\bar{M}_{0, r}(\mathbb{R}) \times \prod_{k} \bar{M}_{0,\left|\lambda_{k}\right|+1}(\mathbb{R})$ into $\bar{M}_{0, \widetilde{r}}(\mathbb{R})$ as follows: take $\left(C, C_{1}, \ldots, C_{r}\right) \in \bar{M}_{0, r}(\mathbb{R}) \times$ $\prod_{k} \bar{M}_{0,\left|\lambda_{k}\right|+1}(\mathbb{R})$ and send it to the stable curve in $\bar{M}_{0, \widetilde{r}}(\mathbb{R})$, where we glue the $\left(\left|\lambda_{k}\right|+1\right)$ th marked point of $C_{k}$ to the $k$ th marked point of $C$.

Look at the family $\mathcal{S}(\square, \square, \ldots, \square)$ over $\bar{M}_{0, \widetilde{r}}(\mathbb{R})$. Restrict it to a family $\mathcal{X}$ over $\bar{M}_{0, r}(\mathbb{R}) \times$ $\prod_{k} \bar{M}_{0,\left|\lambda_{k}\right|+1}(\mathbb{R})$. In the family $\mathcal{X}$, the markings on the $r$ nodes of the central component are locally constant. Let $\mathcal{Y}$ be the union of those components of $\mathcal{X}$ where the markings on the central nodes are $\left(\lambda_{1}, \lambda_{2}, \ldots, \lambda_{r}\right)$.

Take the face $\sigma$ of $\bar{M}_{0, r}$ corresponding to the circular ordering $(1,2, \ldots, r)$. Let $\widetilde{\sigma}$ be some maximal face of $\bar{M}_{0, \widetilde{r}}$ containing $\sigma \times \prod_{k} \bar{M}_{0,\left|\lambda_{k}\right|+1}(\mathbb{R})$. Let $\tau$ in $\mathcal{Y}$ lie over $\sigma$ and let $\widetilde{\tau}$ be the face over $\widetilde{\sigma}$ in $\mathcal{S}(\square, \square, \cdots, \square)$ containing $\tau \times \prod_{k} \bar{M}_{0,\left|\lambda_{k}\right|+1}(\mathbb{R})$ in its boundary. Then $\tau$ is associated to some cylindrical growth pattern $\widetilde{\gamma}$. Restricting $\widetilde{\gamma}$ to $\mathbb{I}$ gives a decgd pattern $\gamma$ of shape $\left(\lambda_{1}, \ldots, \lambda_{r}\right)$.

Theorem 9.2. With the above notations, $\gamma$ depends only on $\tau$, not on the choice of $\widetilde{\tau}$. This gives a bijection between faces of $\mathcal{S}\left(\lambda_{1}, \ldots, \lambda_{r}\right)$ over $\sigma$ and dual equivalence cylindrical growth patterns of shape $\left(\lambda_{1}, \ldots, \lambda_{r}\right)$.

REMARK 9.3. We describe faces of $\mathcal{S}\left(\lambda_{1}, \ldots, \lambda_{r}\right)$ as boundaries of faces of the space $\mathcal{S}(\square, \square, \cdots, \square)$. This is closely related to Sections 5 and 6 of [Pur10], which describe a limiting procedure where marked points on $\mathbb{P}^{1}$ collide. Because we let our points travel through $\bar{M}_{0, d(n-d)}$, rather than $\left(\mathbb{P}^{1}\right)^{d(n-d)}$, we can see precisely where the different dual equivalent growth diagrams wind up in this limiting process - in the different parts of the $\prod \bar{M}_{0,\left|\lambda_{k}\right|+1}(\mathbb{R})$-factor.

Proof. By Proposition 9.1, we know that we can lift $\gamma$ to a cylindrical growth diagram $\widetilde{\gamma}$. Therefore, we have shown that for every dual equivalence cylindrical growth diagram $\gamma$, there is a face $\widetilde{\tau}$ in $\mathcal{S}(\square, \square, \ldots, \square)$ which will give $\gamma$. Our next goal is to show that $\gamma$ depends only on $\tau$ (the face of $\mathcal{Y}$ ), and not on $\widetilde{\tau}$ (the face of $\mathcal{S}(\square, \cdots, \square)$ ).

Let $\widetilde{\tau}$ and $\widetilde{\tau}^{\prime}$ be two faces containing $\tau$; let $\widetilde{\gamma}$ and $\widetilde{\gamma}^{\prime}$ be the corresponding cylindrical growth patterns. Let $\widetilde{a}(k, \ell)$ be the tableau $\left(\widetilde{\gamma}_{\iota(k), \iota(\ell)}, \widetilde{\gamma}_{\iota(k), \iota(\ell)+1}, \ldots, \widetilde{\gamma}_{\iota(k), \iota(\ell+1)}\right)$, let $\widetilde{b}(k, \ell)$ be the tableau $\left(\widetilde{\gamma}_{\iota(k), \iota(\ell)}, \widetilde{\gamma}_{\iota(k)-1, \iota(\ell)}, \ldots, \widetilde{\gamma}_{\iota(k-1), \iota(\ell)}\right)$ and define the primed objects similarly. We want to show that $\widetilde{a}(k, \ell)$ is dual equivalent to $\widetilde{a}^{\prime}(k, \ell)$, and the same for $\widetilde{b}(k, \ell)$ and $\widetilde{b}^{\prime}(k, \ell)$.

We abbreviate $\mathcal{S}(\square, \square, \ldots, \square)$ to $\mathcal{S}$. Let $\mathcal{N}$ be a tubular neighborhood of $\tau \times \prod_{k} \bar{M}_{0,\left|\lambda_{k}\right|+1}(\mathbb{R})$ and let $\pi$ be the projection from $\mathcal{N}$ onto $\tau \times \prod_{k} \bar{M}_{0,\left|\lambda_{k}\right|+1}(\mathbb{R})$. Take a path $p(t)$ from $\widetilde{\tau}$ to $\widetilde{\tau}^{\prime}$ through $\mathcal{N}$ which only passes through cells of codimension 0 or 1 . We are using that $\prod_{k} \bar{M}_{0,\left|\lambda_{k}\right|+1}(\mathbb{R})$ is connected and $\mathcal{S}$ is a manifold. Each maximal cell through which $p(t)$ passes is labeled by a cylindrical growth diagram. We claim that all of these diagrams are dual equivalent.

There are two types of walls we cross through. One type of wall is when $\pi(p(t))$ crosses a wall within one of the factors $\bar{M}_{0,\left|\lambda_{m}\right|+1}(\mathbb{R})$. The edge labels $\widetilde{a}(k, \ell)$ and $\widetilde{b}(k, \ell)$ are unchanged when $k \leqslant m-1<m \leqslant \ell$. Look at the path $\widetilde{b}(m, m), \widetilde{b}(m-1, m), \ldots, \widetilde{b}(m-r, m)$. The tableaux after the first position are preserved; the tableau in the first position is replaced by another one of the 


\section{DAVID E SPEYER}

same straight shape. So, by Proposition 8.2, we have preserved dual equivalence classes along this path and hence in the whole growth diagram.

The other type of wall is one where $\pi(p(t))$ remains in the same cell of $\tau \times \prod_{k} \bar{M}_{0,\left|\lambda_{k}\right|+1}(\mathbb{R})$. When we cross such a wall, we reverse the circular ordering of some interval of points of length $\lambda_{m}$, and the effect on the growth diagram is to switch $\widetilde{a}(m-1, m-1)$ with $\widetilde{b}(m, m)$, while preserving $\widetilde{a}(k, \ell)$ and $\widetilde{b}(k, \ell)$ for $k \leqslant m-1<m \leqslant \ell$. As before, the dual equivalence classes of all the tableaux on the path $\widetilde{b}(m, m), \widetilde{b}(m-1, m), \ldots, \widetilde{b}(m-r, m)$ are unchanged. Again, the dual equivalence class of the cylindrical growth diagram is unchanged in this case.

We have now shown that the map from faces $\tau$ to dual equivalence cylindrical growth diagrams is well defined. We must show that it is bijective. Since we can always lift a decgd $\gamma$ to a cylindrical growth diagram $\widetilde{\gamma}$, the map is surjective.

The covering $\mathcal{Y} \rightarrow \bar{M}_{0, r}$ is of degree $c_{\lambda_{1} \lambda_{2} \cdots \lambda_{r}}$. This is also the number of dual equivalence cylindrical growth diagrams. So the correspondence between maximal faces and decgds is bijective.

We can now describe the $C W$-structure on $\mathcal{S}\left(\lambda_{1}, \lambda_{2}, \ldots, \lambda_{r}\right)$. Its facets are indexed by pairs of a circular ordering of $[r]$ and a dual equivalence cylindrical growth diagram of shape $\left(\lambda_{1}, \ldots, \lambda_{r}\right)$. Finally, we describe how these facets are glued together, generalizing Proposition 7.6.

Let $1 \leqslant p<q \leqslant r$ and suppose we cross a wall reversing the order of $s(q), s(q+1), \ldots$, $s(p-1)$ (indices cyclic modulo $r)$. Let $(\gamma, a, b)$ label the face on one side of the wall and $(\hat{\gamma}, \hat{a}, \hat{b})$ label the face on the other.

For $p \leqslant k \leqslant \ell \leqslant q$, we have $\gamma_{k \ell}=\hat{\gamma}_{k \ell}$; for $p \leqslant k \leqslant \ell<q$, we have $a(k, \ell)=\hat{a}(k, \ell)$; for $p<k \leqslant \ell \leqslant q$, we have $b(k, \ell)=\hat{b}(k, \ell)$. For $q \leqslant k \leqslant \ell \leqslant p+r$, we have $\gamma_{k \ell}=\hat{\gamma}_{(p+q-\ell-1)(p+q-k-1)}$; for $q \leqslant k \leqslant \ell<p+r$, we have $a(k, \ell)=\hat{b}(p+q-\ell-1, p+q-k-1)$ and $\hat{a}(k, \ell)=b(p+q-\ell-$ $1, p+q-k-1)$,

To prove this, simply lift $\gamma$ to some $\widetilde{\gamma}$, cross the corresponding wall in $\mathcal{S}(\square, \square, \cdots, \square)$, and apply Proposition 7.6.

\section{REFERENCES}

Bri02 M. Brion, Positivity in the Grothendieck group of complex flag varieties, J. Algebra 258 (2002), no. 1, 137-159. http://dx.doi.org/10.1016/S0021-8693(02)00505-7

Dev04 S. Devadoss, Combinatorial equivalence of real moduli spaces, Notices Amer. Math. Soc. 51 (2004), no. 6, 620-628.

EH83 D. Eisenbud and J. Harris, Divisors on general curves and cuspidal rational curves, Invent. Math. 74 (1983), no. 3, 371-418.

EH87 D. Eisenbud and J. Harris, Limit linear series: basic theory, Invent. Math. 85 (1986), no. 2, 337-371.

EG02 A. Eremenko and A. Gabrielov, Rational functions with real critical points and the B. and $M$. Shapiro conjecture in real enumerative geometry, Ann. of Math. (2) 155 (2002), no. 1, 105-129. http://dx.doi.org/10.2307/3062151

Hai92 M. Haiman, Dual equivalence with applications, including a conjecture of Proctor, Discrete Math. 99 (1992), no. 1-3, 79-113.

HK06 A. Henriques and J. Kamnitzer, The octahedron recurrence and $\mathfrak{g l}(n)$ crystals, Adv. Math. 206 (2006), no. 1, 211-249. http://dx.doi.org/10.1016/j.aim.2005.08.007

Kap93 M. M. Kapranov, Veronese curves and Grothendieck-Knudsen moduli space $\bar{M}_{0, n}$, J. Algebraic Geom. 2 (1993), no. 2, 239-262. 


\section{Schubert PROBlems From STABle CURVES}

KK99 M. Khovanov and G. Kuperberg, Web bases for sl(3) are not dual canonical, Pacific J. Math. 188 (1999), no. 1, 129-153.

KTW04 A. Knutson, T. Tao and C. Woodward, A positive proof of the Littlewood-Richardson rule using the octahedron recurrence, Electron. J. Combin. 11 (2004), no. 1, Research Paper 61

Kup96 G. Kuperberg, Spiders for rank 2 Lie algebras, Comm. Math. Phys. 180 (1996), no. 1, 109-151.

Mat86 H. Matsumura, Commutative Ring Theory, Translated from the Japanese by M. Reid, Cambridge Studies in Advanced Mathematics, 8, Cambridge University Press, Cambridge, 1986.

MTV09 E. Mukhin, V. Tarasov and A. Varchenko, The B. and M. Shapiro conjecture in real algebraic geometry and the Bethe ansatz, Ann. of Math. (2) 170 (2009), no. 2, 863-881. http://dx.doi. org/10.4007/annals.2009.170.863

PPR09 T. K. Petersen, P. Pylyavskyy and B. Rhoades, Promotion and cyclic sieving via webs, J. Algebraic Combin. 30 (2009), no. 1, 19-41. http://dx.doi.org/10.1007/s10801-008-0150-3

Pur10 K. Purbhoo, Jeu de taquin and a monodromy problem for Wronskians of polynomials, Adv. Math. 224 (2010), no. 3, 827-862. http://dx.doi.org/10.1016/j.aim.2009.12.013

Pur13 K. Purbhoo, Wronskians, cyclic group actions, and ribbon tableaux, Trans. Amer. Math. Soc. 365 (2013), no. 4, 1977-2030. http://dx.doi.org/10.1090/S0002-9947-2012-05676-5

Ram85 A. Ramanathan, Schubert varieties are arithmetically Cohen-Macaulay, Invent. Math. 80 (1985), no. 2, 283-294.

RSSS06 J. Ruffo, Y. Sivan, E. Soprunova and F. Sotille, Experimentation and Conjectures in the Real Schubert Calculus for Flag Manifolds, Experiment. Math. 15 (2006), no. 2, 199-221. http: //dx.doi.org/10.1080/10586458.2006.10128954

Sta99 R. P. Stanley, Enumerative Combinatorics 2 Cambridge Studies in Advanced Mathematics, 62 Cambridge University Press, Cambridge, 1999.

TY08 T. Thomas and A. Yong, An $S_{3}$-symmetric Littlewood-Richardson rule, Math. Res. Lett. 15 (2008), no. 5, 1027-1037. http://dx.doi.org/10.4310/MRL.2008.v15.n5.a15

David E Speyer speyer@umich.edu

Department of Mathematics, 2844 East Hall, 530 Church Street, Ann Arbor, MI 48109, USA 